\title{
Toward GIT stability of syzygies of canonical curves
}

\author{
Anand Deopurkar, Maksym Fedorchuk and David Swinarski
}

\begin{abstract}
We introduce the problem of GIT stability for syzygy points of canonical curves with a view toward a GIT construction of the canonical model of $\bar{M}_{g}$. As the first step in this direction, we prove the semi-stability of the first syzygy point for a general canonical curve of odd genus.
\end{abstract}

\section{Introduction}

By analogy with Hilbert points, as introduced by Gieseker in [Gie77, $§ 1$ ], we introduce syzygy points of canonical curves and initiate a program of studying their geometric invariant theory (GIT) stability. The eventual goal of this program is a GIT construction of the canonical model of $\bar{M}_{g}$, a problem whose origins lie in the log minimal model program for the moduli space of stable curves. Introduced by Hassett and Keel, the log minimal model program for $\bar{M}_{g}$ aims to construct certain log canonical models of $\bar{M}_{g}$ in a way that allows modular interpretation [Has05]. The log canonical divisors on (the stack) $\overline{\mathcal{M}}_{g}$ considered in this program are

$$
K_{\overline{\mathcal{M}}_{g}}+\alpha \delta=13 \lambda-(2-\alpha) \delta, \quad \text { where } \quad \alpha \in[0,1] \cap \mathbb{Q} .
$$

The work done so far suggests that we can construct some of these models as GIT quotients of spaces of Hilbert points of $n$-canonically embedded curves. This is already evidenced in the work of Gieseker [Gie82] and Schubert [Sch91b], who analyzed the cases $n \geqslant 5$ and $n=3$, respectively. Recent work of Hassett and Hyeon [HH09, HH13] extends the GIT analysis to $n=2$ and constructs the $\log$ canonical models of $\bar{M}_{g}$ corresponding to $\alpha>7 / 10-\varepsilon$; see also [AFSvdW13] for a non-GIT construction of the models corresponding to $\alpha>2 / 3-\varepsilon$. Subsequent work along this direction suggests that the case $n=1$ and the use of finite Hilbert points would yield log canonical models corresponding to the values of $\alpha$ down to $\alpha=(g+6) /(7 g+6)$ [AFS13, FJ13].

The ultimate goal of the Hassett-Keel program is to reach $\alpha=0$, which corresponds to the canonical model of $\bar{M}_{g}$. To go beyond $\alpha=(g+6) /(7 g+6)$ and indeed down to $\alpha=0$, Farkas and Keel suggested that one should construct birational models of $\bar{M}_{g}$ by performing GIT analysis on syzygies of canonically embedded curves. In this paper, we make the first step toward this goal by proving a generic semi-stability result for the first syzygies in odd genus.

Main Theorem. A general canonical curve of odd genus $g \geqslant 5$ has a semi-stable first syzygy point.

Received 23 February 2014, accepted in final form 21 March 2015.

2010 Mathematics Subject Classification 14L24, 13D02, $14 \mathrm{H} 10$.

Keywords: canonical curves, syzygies, geometric invariant theory, ribbons.

This journal is (C) Foundation Compositio Mathematica 2016. This article is distributed with Open Access under the terms of the Creative Commons Attribution Non-Commercial License, which permits non-commercial reuse, distribution, and reproduction in any medium, provided that the original work is properly cited. For commercial re-use, please contact the Foundation Compositio Mathematica.

During the preparation of this article the second author was partially supported by the NSF grant DMS-1259226 and a Sloan Research Fellowship. 


\section{A. Deopurkar, M. Fedorchuk and D. Swinarski}

Our strategy for proving the generic semi-stability of syzygy points follows that of [AFS13] for proving the generic semi-stability of finite Hilbert points. Namely, we prove the semi-stability of the first syzygy point of a singular curve with $\mathbb{G}_{m}$-action, the balanced ribbon, by a method suggested by Morrison and Swinarski in [MS11].

Ribbons and the problem of studying syzygies of their canonical embeddings were originally introduced by Bayer and Eisenbud in [BE95]. Their motivation for studying ribbons was in the context of Green's conjecture for smooth canonical curves. Namely, Bayer and Eisenbud asked whether rational ribbons satisfy an appropriate version of Green's conjecture [BE95, Introduction]. Although this question remains open in its full generality, an affirmative answer to it implies the generic Green's conjecture, which is known thanks to the work of Voisin [Voi02, Voi05]. Green's conjecture makes an appearance in the study of GIT stability of syzygies of canonical curves by controlling which syzygy points are well defined; see Remark 2.4.

Outline of the paper. In Section 2, we define syzygy points of a canonically embedded Gorenstein curve and give a precise statement of our main result. In Section 3, we recall some preliminary results about balanced ribbons. In the most technical section, Section 4, we construct several monomial bases of cosyzygies for the balanced ribbon. Finally, in Section 5, we prove the main theorem by deducing the semi-stability of the first syzygy point of the balanced ribbon from the existence of the monomial bases constructed in Section 4.

\section{Syzygy points of canonical curves}

In this section, we recall some basic notions of Koszul cohomology and set up GIT problems for the linear syzygies of a canonical curve. We refer to [Gre84] and [AF11b] for a complete treatment of Koszul cohomology and a detailed discussion of Green's conjecture.

We define a canonical Gorenstein curve to be a complete Gorenstein curve $C$ with a very ample dualizing sheaf $\omega_{C}$. The arithmetic genus of such $C$ is at least 3 . In this paper, we are exclusively concerned with Koszul cohomology of the pair $\left(C, \omega_{C}\right)$. Namely, associated to $C$ and the line bundle $\omega_{C}$ is the Koszul complex

$$
\bigwedge^{p+1} \mathrm{H}^{0}\left(\omega_{C}\right) \otimes \mathrm{H}^{0}\left(\omega_{C}^{q-1}\right) \stackrel{f_{p+1, q-1}}{\longrightarrow} \bigwedge^{p} \mathrm{H}^{0}\left(\omega_{C}\right) \otimes \mathrm{H}^{0}\left(\omega_{C}^{q}\right) \stackrel{f_{p, q}}{\longrightarrow} \bigwedge_{p-1}^{\longrightarrow} \mathrm{H}^{0}\left(\omega_{C}\right) \otimes \mathrm{H}^{0}\left(\omega_{C}^{q+1}\right),
$$

where the differential $f_{p, q}$ is given by

$$
f_{p, q}\left(x_{0} \wedge x_{1} \wedge \cdots \wedge x_{p-1} \otimes y\right)=\sum_{i=0}^{p-1}(-1)^{i} x_{0} \wedge \cdots \wedge \widehat{x}_{i} \wedge \cdots \wedge x_{p-1} \otimes x_{i} y .
$$

The Koszul cohomology groups of $\left(C, \omega_{C}\right)$ are

$$
K_{p, q}(C):=\operatorname{ker} f_{p, q} / \operatorname{im} f_{p+1, q-1} .
$$

We say that $C$ satisfies property $\left(N_{p}\right)$ if $K_{i, q}(C)=0$ for all $(i, q)$ with $i \leqslant p$ and $q \geqslant 2$. In particular, property $\left(N_{0}\right)$ means that the natural maps $\operatorname{Sym}^{m} \mathrm{H}^{0}\left(\omega_{C}\right) \rightarrow \mathrm{H}^{0}\left(\omega_{C}^{m}\right)$ are surjective for all $m$, or, equivalently, that $C$ is projectively normal in its canonical embedding. Property $\left(N_{p}\right)$ for $p \geqslant 1$ means, in addition, that the ideal of $C$ in the canonical embedding is generated by quadrics and the syzygies of order up to $p$ are linear.

Remark 2.1 (On the projective normality of canonical Gorenstein curves). A classical theorem of Max Noether says that a smooth curve $C$ of genus $g \geqslant 3$ is non-hyperelliptic if and only if $\omega_{C}$ is 
very ample if and only if $C$ satisfies property $\left(N_{0}\right)$ [ACGH85, Chapter III, $\left.\S 2\right]$. A relatively recent result [KM09] extends these equivalences to the case when $C$ is an integral Gorenstein curve. We are not aware of a general result concerning projective normality of non-integral Gorenstein curves. In particular, it appears that the case of reducible curves is open in general [BB11]. Because of this and because our primary object of study is a non-reduced curve (namely, a rational ribbon), we often specify projective normality as a hypothesis. We do note that projective normality of non-hyperelliptic ribbons is established in the original paper of Bayer and Eisenbud [BE95, Theorem 5.3]. An explicit proof for the case of the balanced ribbon appears in [AFS13, Proposition 3.5].

Let $C$ be a canonical Gorenstein curve of genus $g \geqslant 3$ satisfying property $\left(N_{0}\right)$. Then the canonical embedding of $C$ is an arithmetically Gorenstein curve in $\mathbb{P}^{g-1}$, and, from the self-duality of the minimal projective resolution of its homogeneous coordinate ring, we have $K_{p, q}(C)=0$ for all $q \geqslant 3$ and $p \leqslant g-3$; see [Sch91a, Corollary 1.3]. In particular, for all $p<\lfloor g / 2\rfloor$, property $\left(N_{p}\right)$ is equivalent to $K_{p, 2}(C)=0$. For $p \leqslant g-2$, set

$$
\Gamma_{p}(C):=\left(\bigwedge^{p+1} \mathrm{H}^{0}\left(\omega_{C}\right) \otimes \mathrm{H}^{0}\left(\omega_{C}\right)\right) / \stackrel{p+2}{\bigwedge} \mathrm{H}^{0}\left(\omega_{C}\right) .
$$

We can readily compute that

$$
\begin{aligned}
\operatorname{dim} \operatorname{ker} f_{p, 2} & =(3 g-2 p-3)\left(\begin{array}{c}
g-1 \\
p
\end{array}\right) \text { and } \\
\operatorname{dim} \Gamma_{p}(C) & =g\left(\begin{array}{c}
g \\
p+1
\end{array}\right)-\left(\begin{array}{c}
g \\
p+2
\end{array}\right) .
\end{aligned}
$$

The first four terms of the Koszul complex (2.1) in degree $p+2$ give the exact sequence

$$
0 \rightarrow K_{p+1,1}(C) \rightarrow \Gamma_{p}(C) \rightarrow \operatorname{ker} f_{p, 2} \rightarrow K_{p, 2}(C) \rightarrow 0 .
$$

Definition 2.2. We define the space of pth-order linear syzygies of $C$ as the subspace of $\Gamma_{p}(C)$ given by

$$
\operatorname{Syz}_{p}(C):=K_{p+1,1}(C) .
$$

Suppose that $C$ satisfies property $\left(N_{p}\right)$, so that $K_{p, 2}(C)=0$. We define the space of pth-order linear cosyzygies of $C$ as the quotient space of $\Gamma_{p}(C)$ given by

$$
\operatorname{CoSyz}_{p}(C):=\operatorname{ker} f_{p, 2} \text {. }
$$

The relation of the above definition to the definition of syzygies in terms of the homogeneous ideal of $C$ is as follows. Let

$$
I_{m}(C)=\operatorname{ker}\left(\operatorname{Sym}^{m} \mathrm{H}^{0}\left(\omega_{C}\right) \rightarrow \mathrm{H}^{0}\left(\omega_{C}^{m}\right)\right)
$$

be the degree $m$ graded piece of the homogeneous ideal of $C$. Then the space of $p$ th-order linear syzygies among the defining quadrics of $C$ is taken to be the kernel of the map

$$
\bigwedge^{p} \mathrm{H}^{0}\left(\omega_{C}\right) \otimes I_{2}(C) \stackrel{\gamma}{\longrightarrow} \bigwedge^{p-1} \mathrm{H}^{0}\left(\omega_{C}\right) \otimes I_{3}(C)
$$

A simple diagram chase now gives a well-known isomorphism $\operatorname{ker} \gamma \simeq K_{p+1,1}(C)$. 


\section{A. Deopurkar, M. Fedorchuk and D. Swinarski}

Definition 2.3. Suppose that $C$ satisfies property $\left(N_{p}\right)$. We define the pth syzygy point of $C$ to be the quotient of $\Gamma_{p}(C)$ given by

$$
\left[\Gamma_{p}(C) \rightarrow \operatorname{CoSyz}_{p}(C) \rightarrow 0\right]
$$

and interpreted as a point in the Grassmannian $\operatorname{Grass}\left((3 g-2 p-3)\left(\begin{array}{c}g-1 \\ p\end{array}\right), \Gamma_{p}(C)\right)$.

Abusing notation, we use $\operatorname{CoSyz}_{p}(C)$ to denote both the vector space itself and the point in Grass $\left((3 g-2 p-3)\left(\begin{array}{c}g-1 \\ p\end{array}\right), \Gamma_{p}(C)\right)$ that it represents. Observe that the zeroth syzygy point is simply the second Hilbert point.

Remark 2.4. For which curves is the $p$ th syzygy point defined? According to a celebrated conjecture, a smooth canonical curve $C$ satisfies $\left(N_{p}\right)$ if and only if $p$ is less than the Clifford index of $C$. Formulated by Green in [Gre84], this conjecture remains open in its full generality. It is known to be true, however, for a large class of curves. Voisin proved that general canonical curves on $K 3$ surfaces satisfy Green's conjecture [Voi02, Voi05]. More recently, Aprodu and Farkas proved the conjecture for all smooth curves on $K 3$ surfaces [AF11a]. In particular, the $p$ th syzygy point of a generic curve of genus $g$ is defined for all $p<\lfloor g / 2\rfloor$.

Definition 2.5. We define $\overline{\mathrm{Syz}}_{p}$ to be the closure in $\operatorname{Grass}\left((3 g-2 p-3)\left(\begin{array}{c}g-1 \\ p\end{array}\right), \Gamma_{p}(C)\right)$ of the locus of $p$ th syzygy points of canonical curves satisfying property $\left(N_{p}\right)$.

Consider the group $\mathrm{SL}_{g} \simeq \mathrm{SL}\left(\mathrm{H}^{0}\left(\omega_{C}\right)\right)$. Its natural action on $\mathrm{H}^{0}\left(\omega_{C}\right)$ induces actions on the vector space $\Gamma_{p}(C)$, the Grassmannian Grass $\left((3 g-2 p-3)\left(\begin{array}{c}g-1 \\ p\end{array}\right), \Gamma_{p}(C)\right)$, and finally on the subvariety $\overline{\mathrm{Syz}}_{p}$. The Plücker line bundle on the Grassmannian is equipped with a natural $\mathrm{SL}_{g}$ linearization, and so is its restriction to $\overline{\mathrm{Syz}}_{p}$. A candidate for the pth syzygy model of $\bar{M}_{g}$ is thus the GIT quotient

$$
\overline{\mathrm{Syz}}_{p} / / \mathrm{SL}_{g} .
$$

Our main theorem shows that this quotient is non-empty for $p=1$ and odd $g \geqslant 5$.

Theorem 2.6. A general canonical curve of odd genus $g \geqslant 5$ has a semi-stable first syzygy point.

We prove this theorem in Section 5; see Corollary 5.5.

\section{The balanced canonical ribbon}

We prove Theorem 2.6 by explicitly writing down a semi-stable point in $\overline{\mathrm{Syz}}_{1}$. This point corresponds to the syzygies of the balanced ribbon. Our exposition of its properties closely follows [AFS13], where the semi-stability of Hilbert points of this ribbon was established. Nevertheless, we recall the necessary details for the reader's convenience.

Let $g=2 k+1$. The balanced ribbon of genus $g$ is the scheme $R$ obtained by identifying $U:=\operatorname{Spec} \mathbb{C}[u, \epsilon] /\left(\epsilon^{2}\right)$ and $V:=\operatorname{Spec} \mathbb{C}[v, \eta] /\left(\eta^{2}\right)$ along $U \backslash\{0\}$ and $V \backslash\{0\}$ via the isomorphism

$$
\begin{aligned}
u & \mapsto v^{-1}-v^{-k-2} \eta, \\
\epsilon & \mapsto v^{-g-1} \eta .
\end{aligned}
$$

The scheme $R$ is an example of a rational ribbon. While our arguments use only the balanced ribbon, we refer the reader to [BE95] for a more extensive study of ribbons in general. 


\section{TOWARD GIT STABILITY OF SYZYGIES OF CANONICAL CURVES}

Being a Gorenstein curve, $R$ has a dualizing line bundle $\omega$, trivialized by $(d u \wedge d \epsilon) / \epsilon^{2}$ on $U$ and by $(d v \wedge d \eta) / \eta^{2}$ on $V$. Since $\omega$ is very ample by [AFS13, Lemma 3.2], the global sections of $\omega$ embed $R$ as an arithmetically Gorenstein curve in $\mathbb{P}^{g-1}$ by [BE95, Theorem 5.3].

The balanced ribbon $R$ admits a $\mathbb{G}_{m}$-action, given by

$$
\begin{array}{rlrl}
t \cdot u & \mapsto t u, & t \cdot \epsilon & \mapsto t^{k+1} \epsilon, \\
t \cdot v \mapsto t^{-1} v, & t \cdot \eta \mapsto t^{-k-1} \eta .
\end{array}
$$

This action induces $\mathbb{G}_{m}$-actions on $\mathrm{H}^{0}\left(R, \omega^{m}\right)$ for all $m$. The next two propositions describe these spaces along with their decompositions into weight spaces.

Proposition 3.1. A basis for $\mathrm{H}^{0}(R, \omega)$ is given by $x_{0}, \ldots, x_{2 k}$, where the $x_{i}$ restricted to $U$ are given by

$$
x_{i}= \begin{cases}u^{i} \frac{d u \wedge d \epsilon}{\epsilon^{2}} & \text { if } \quad 0 \leqslant i \leqslant k, \\ \left(u^{i}+(i-k) u^{i-k-1} \epsilon\right) \frac{d u \wedge d \epsilon}{\epsilon^{2}} & \text { if } \quad k<i \leqslant 2 k,\end{cases}
$$

and where $x_{i}$ is a $\mathbb{G}_{m}$-semi-invariant of weight $i-k$. In particular, $\mathrm{H}^{0}(R, \omega)$ splits as a direct sum of $g$ distinct $\mathbb{G}_{m}$-weight spaces of weights $-k, \ldots, k$.

Proof. That the $x_{i}$ form a basis follows from [BE95, Theorem 5.1]. The statement about the weights is obvious.

Remark $3.2\left(\mathbb{Z}_{2}\right.$-symmetry). Observe that $R$ has a $\mathbb{Z}_{2}$-symmetry given by the isomorphism $V \simeq U$ defined by $u \leftrightarrow v$ and $\epsilon \leftrightarrow \eta$ and commuting with the gluing isomorphism (3.1). This $\mathbb{Z}_{2^{-}}$ symmetry exchanges $x_{i}$ and $x_{2 k-i}$.

The following observation from [AFS13, Lemma 3.4] deals with higher powers of $\omega$.

Lemma 3.3 (Ribbon product lemma). Let $0 \leqslant i_{1}, \ldots, i_{m} \leqslant 2 k$ be such that $i_{1}, \ldots, i_{\ell} \leqslant k$ and $i_{\ell+1}, \ldots, i_{m}>k$. On $U$, we have

$$
x_{i_{1}} \cdots x_{i_{m}}=\left(u^{a}+(a-b) u^{a-k-1} \epsilon\right)\left(\frac{d u \wedge d \epsilon}{\epsilon^{2}}\right)^{m},
$$

where

$$
\begin{aligned}
& a=i_{1}+\cdots+i_{m}, \\
& b=i_{1}+\cdots+i_{\ell}+k(m-\ell) .
\end{aligned}
$$

Definition 3.4. The $u$-weight (or $u$-degree) of a monomial $x_{i_{1}} \cdots x_{i_{m}}$ is the sum $i_{1}+\cdots+i_{m}$. Note that the $u$-weight of $x_{i_{1}} \cdots x_{i_{m}}$ equals the $\mathbb{G}_{m}$-weight of $x_{i_{1}} \cdots x_{i_{m}}$ plus $m k$.

Proposition 3.5. Let $m \geqslant 2$. Let $\mathrm{H}^{0}\left(R, \omega^{m}\right)_{d}$ be the weight space of $\mathrm{H}^{0}\left(R, \omega^{m}\right)$ of $u$-weight $d$. Then

$$
\operatorname{dim} \mathrm{H}^{0}\left(R, \omega^{m}\right)_{d}= \begin{cases}1 & \text { if } 0 \leqslant d \leqslant k, \\ 2 & \text { if } k<d<2 m k-k, \\ 1 & \text { if } 2 m k-k \leqslant d \leqslant 2 m k .\end{cases}
$$

Moreover, the map $\operatorname{Sym}^{m} \mathrm{H}^{0}(R, \omega) \rightarrow \mathrm{H}^{0}\left(R, \omega^{m}\right)$ is surjective.

Proof. Using the generator $\left((d u \wedge d \epsilon) / \epsilon^{2}\right)^{m}$ of $\omega^{m}$ on $U$, let us identify the sections of $\omega^{m}$ on $U$ with the elements of $\mathbb{C}[u, \epsilon] /\left(\epsilon^{2}\right)$. Consider the following $(2 m-1)(g-1)$ sections of $\omega^{m}$ on $U$ :

$$
\left\{u^{i}\right\}_{i=0}^{2 m k-k-1}, \quad\left\{u^{i}+(i-m k) u^{i-k-1} \epsilon\right\}_{i=k+1}^{2 m k} .
$$




\section{A. Deopurkar, M. Fedorchuk and D. Swinarski}

We claim that these sections are in the image of $\operatorname{Sym}^{m} \mathrm{H}^{0}(R, \omega)$. Indeed, for $0 \leqslant i \leqslant k$, the monomial $x_{0}^{m-1} x_{i}$ restricts to $u^{i}$ on $U$. For $2 m k-k \leqslant i \leqslant 2 m k$, the monomial $x_{2 k}^{m-1} x_{i+2 k-2 m k}$ restricts to $u^{i}+(i-m k) u^{i-k-1}$. For $k<i<2 m k-k$, it suffices to exhibit two monomials $x_{i_{1}} \cdots x_{i_{m}}$ with $i_{1}+\cdots+i_{m}=i$ whose restrictions to $U$ are linearly independent. This is easy to do using Lemma 3.3; we leave this to the reader.

We conclude that the sections listed in (3.3) extend to global sections of $\omega^{m}$. By construction, these global sections are in the image of $\operatorname{Sym}^{m} \mathrm{H}^{0}(R, \omega)$. Since these sections are linearly independent and their number equals $h^{0}\left(\omega^{m}\right)$, they form a basis of $\mathrm{H}^{0}\left(\omega^{m}\right)$. We conclude that $\operatorname{Sym}^{m} \mathrm{H}^{0}(R, \omega) \rightarrow \mathrm{H}^{0}\left(R, \omega^{m}\right)$ is surjective. The sections $u^{i}\left((d u \wedge d \epsilon) / \epsilon^{2}\right)^{m}$ are semi-invariants of $\mathbb{G}_{m}$ with weights $-m k, \ldots, m k-k-1$. The sections $\left(u^{i}+(i-m k) u^{i-k-1} \epsilon\right)\left((d u \wedge d \epsilon) / \epsilon^{2}\right)^{m}$ are semi-invariants of $\mathbb{G}_{m}$ with weights $-m k+k+1, \ldots, m k$. The claim about the dimensions of the weight spaces follows.

The following is immediate from Proposition 3.5.

Corollary 3.6. Let $\mathcal{B}$ be a set of monomials of degree $m$ in the variables $x_{0}, \ldots, x_{2 k}$. Its image in $\mathrm{H}^{0}\left(R, \omega^{m}\right)$ forms a basis if and only if

(i) for $0 \leqslant d \leqslant k$ and $2 m k-k \leqslant d \leqslant 2 m k$, the set $\mathcal{B}$ contains exactly one monomial of $u$-weight $d$;

(ii) for $k<d<2 m k-k$, the set $\mathcal{B}$ contains exactly two monomials of $u$-weight $d$ and these two monomials are linearly independent in $\mathrm{H}^{0}\left(R, \omega^{m}\right)$.

We recall the following result.

Proposition 3.7. The following are bases of $\mathrm{H}^{0}\left(R, \omega^{2}\right)$ :

$$
\begin{aligned}
\mathcal{B}^{+} & :=\left\{x_{0} x_{i}\right\}_{i=0}^{2 k} \cup\left\{x_{k} x_{i}\right\}_{i=1}^{2 k-1} \cup\left\{x_{2 k} x_{i}\right\}_{i=1}^{2 k}, \\
\mathcal{B}^{-} & :=\left\{x_{i}^{2}\right\}_{i=0}^{2 k} \cup\left\{x_{i} x_{i+1}\right\}_{i=0}^{2 k-1} \cup\left\{x_{i} x_{i+k}\right\}_{i=1}^{k-1} \cup\left\{x_{i} x_{i+k+1}\right\}_{i=0}^{k-1} .
\end{aligned}
$$

Both $\mathcal{B}^{+}$and $\mathcal{B}^{-}$are invariant with respect to the $\mathbb{Z}_{2}$-symmetry of $R$ and consist of $\mathbb{G}_{m}$-semiinvariant sections. The breakdown of $\mathcal{B}^{+}$by $u$-weight in the range $0 \leqslant d \leqslant 2 k$ is as follows:

$$
\begin{array}{ll}
x_{0} x_{d} & \text { for } 0 \leqslant d \leqslant k, \\
x_{0} x_{d}, x_{k} x_{d-k} & \text { for } k<d \leqslant 2 k .
\end{array}
$$

The breakdown of $\mathcal{B}^{-}$by $u$-weight in the range $0 \leqslant d \leqslant 2 k$ is as follows:

$$
\begin{aligned}
& x_{\lfloor d / 2\rfloor} x_{\lceil d / 2\rceil} \quad \text { for } 0 \leqslant d \leqslant k \text {, } \\
& x_{\lfloor d / 2\rfloor} x_{\lceil d / 2\rceil}, x_{\lfloor(d-k) / 2\rfloor} x_{\lceil(d+k) / 2\rceil} \text { for } k<d \leqslant 2 k .
\end{aligned}
$$

The breakdowns in the range $2 k \leqslant d \leqslant 4 k$ are obtained by using the $\mathbb{Z}_{2}$-symmetry.

Proof. The fact that $\mathcal{B}^{+}$and $\mathcal{B}^{-}$are bases of $\mathrm{H}^{0}\left(R, \omega^{2}\right)$ is the content of [AFS13, Lemma 4.3]. The weight-decomposition statement is obvious.

We record a simple observation about expressing arbitrary quadratic monomials in $\mathrm{H}^{0}\left(R, \omega^{2}\right)$ in terms of the monomials of $\mathcal{B}^{-}$that will be used repeatedly in Subsection 4.2.

Lemma 3.8 (Quadratic equations). Consider $0 \leqslant i \leqslant j \leqslant 2 k$ and set $d=i+j$. Then in $\mathrm{H}^{0}\left(R, \omega^{2}\right)$ we have a relation

$$
x_{i} x_{j}=\lambda x_{\lfloor d / 2\rfloor} x_{\lceil d / 2\rceil}+\mu x_{\lfloor(d-k) / 2\rfloor} x_{\lceil(d+k) / 2\rceil},
$$




\section{TOWARD GIT STABILITY OF SYZYGIES OF CANONICAL CURVES}

where $\lambda$ and $\mu$ are uniquely determined rational numbers. In addition, $\lambda$ and $\mu$ satisfy

(i) $\lambda+\mu=1$;

(ii) if $j \leqslant k$ or $i \geqslant k$, then $\mu=0$;

(iii) if $j-i=k$ or $j-i=k+1$, then $\lambda=0$;

(iv) if $j-i<k$, then $\lambda, \mu>0$;

(v) if $j-i>k+1$, then $\lambda<0, \mu>0$.

Proof. The existence and uniqueness of the relation follow from Proposition 3.7. We now establish the claims about the coefficients for $k<d<3 k$, the remaining cases being clear. By the $\mathbb{Z}_{2^{-}}$ symmetry, we may take $k<d \leqslant 2 k$. If $j \leqslant k$, the statement is clear. If $j>k$, then

$$
\begin{aligned}
x_{\lfloor d / 2\rfloor} x_{\lceil d / 2\rceil} & =u^{d}, \\
x_{\lfloor(d-k) / 2\rfloor} x_{\lceil(d+k) / 2\rceil} & =u^{d}+\lceil(d-k) / 2\rceil u^{d-k-1} \epsilon, \\
x_{i} x_{j} & =u^{d}+(j-k) u^{d-k-1} \epsilon .
\end{aligned}
$$

Now, claim (i) follows by equating the coefficients of $u^{d}$. If $j-i=k$ or $j-i=k+1$, then $(i, j)=(\lfloor(d-k) / 2\rfloor,\lceil(d+k) / 2\rceil)$, so claim (iii) follows. Finally, claims (iv) and (v) follow by equating the coefficients of $u^{d-k-1} \epsilon$ and observing that if $j-i<k$, then $j-k<\lceil(d-k) / 2\rceil$, and if $j-i>k+1$, then $j-k>\lceil(d-k) / 2\rceil$.

\section{Monomial bases of cosyzygies}

In this technical heart of the paper, we introduce monomial bases of cosyzygies for a canonical Gorenstein curve $C$. These correspond to Plücker coordinates of the first syzygy point of $C$ with respect to a fixed basis of $\mathrm{H}^{0}\left(C, \omega_{C}\right)$ and thus can be used in verifying the semi-stability of the first syzygy point of $C$ via the Hilbert-Mumford numerical criterion. We then construct three particular monomial bases of cosyzygies $\mathcal{C}^{+}, \mathcal{C}^{-}$, and $\mathcal{C}^{\star}$ for the balanced ribbon $R$. These bases will be used in the proof of Theorem 5.4. These constructions are done in Subsections 4.1, 4.2, and 4.3 , respectively.

Let $C$ be a canonical Gorenstein curve of genus $g$. Set $\mathrm{H}^{0}(\omega):=\mathrm{H}^{0}\left(C, \omega_{C}\right)$, and let $\Gamma:=\Gamma_{1}(C)$ be as defined in (2.2). For the first syzygy point, the relevant strand of the Koszul complex is

$$
0 \rightarrow \Gamma \stackrel{f_{2,1}}{\longrightarrow} \mathrm{H}^{0}(\omega) \otimes \mathrm{H}^{0}\left(\omega^{2}\right) \stackrel{f_{1,2}}{\longrightarrow} \mathrm{H}^{0}\left(\omega^{3}\right) \rightarrow 0 .
$$

Fix a basis $\left\{x_{0}, \ldots, x_{g-1}\right\}$ of $\mathrm{H}^{0}(\omega)$. For $x, y, z \in\left\{x_{0}, \ldots, x_{g-1}\right\}$, we call the image of $(x \wedge y) \otimes z$ in $\Gamma$ a cosyzygy. With this convention, the only linear relations among cosyzygies in $\Gamma$ are

$$
(x \wedge y) \otimes z+(y \wedge z) \otimes x+(z \wedge x) \otimes y=0 .
$$

By Definition 2.3, the first syzygy point of $C$ is well defined if and only if $K_{1,2}(C)=0$ if and only if the map $\Gamma \rightarrow \operatorname{ker} f_{1,2}$ given by (4.1) is surjective.

Definition 4.1. A set $\mathcal{C}=\left\{\left(x_{a} \wedge x_{b}\right) \otimes x_{c}\right\}_{(a, b, c) \in S} \subset \Gamma$ is called a monomial basis of cosyzygies if the set $\left\{f_{2,1}\left(\left(x_{a} \wedge x_{b}\right) \otimes x_{c}\right)\right\}_{(a, b, c) \in S}$ forms a basis of ker $f_{1,2}$.

Suppose $K_{1,2}(C)=0$, so that the first syzygy point $\operatorname{CoSyz}_{1}(C)$ is well defined. Let $T \subset$ $\mathrm{SL}\left(\mathrm{H}^{0}(\omega)\right)$ be the maximal torus acting diagonally on the fixed basis $\left\{x_{0}, \ldots, x_{g-1}\right\}$ of $\mathrm{H}^{0}(\omega)$. Then the monomial bases of cosyzygies of $C$ correspond precisely to the non-zero Plücker coordinates of $\operatorname{CoSyz}_{1}(C) \in \operatorname{Grass}((3 g-5)(g-1), \Gamma)$ with respect to the distinguished basis of $\Gamma$ 


\section{A. Deopurkar, M. Fedorchuk and D. Swinarski}

consisting of the $T$-eigenvectors. To every such coordinate, and in turn, to every monomial basis of cosyzygies $\mathcal{C}$, we can associate a $T$-character, called the $T$-state of $\mathcal{C}$, which is represented by a linear combination of $x_{0}, \ldots, x_{g-1}$. Precisely, the $T$-state of $\mathcal{C}=\left\{\left(x_{a} \wedge x_{b}\right) \otimes x_{c}\right\}_{(a, b, c) \in S}$ is given by

$$
w_{T}(\mathcal{C}):=\sum_{(a, b, c) \in S} w_{T}\left(\left(x_{a} \wedge x_{b}\right) \otimes x_{c}\right)=\sum_{(a, b, c) \in S}\left(x_{a}+x_{b}+x_{c}\right)=n_{0} x_{0}+\cdots+n_{g-1} x_{g-1},
$$

where $n_{i}$ is the number of occurrences of $x_{i}$ among the cosyzygies in $\mathcal{C}$. Note that we always have

$$
\sum_{i=0}^{g-1} n_{i}=3(3 g-5)(g-1) .
$$

Remark 4.2. Recall from equation (2.3) that

$$
\operatorname{dim} \operatorname{ker} f_{1,2}=(3 g-5)(g-1) .
$$

Therefore, a set $\mathcal{C}=\left\{\left(x_{a} \wedge x_{b}\right) \otimes x_{c}\right\}_{(a, b, c) \in S} \subset \Gamma$ is a monomial basis of cosyzygies if and only if the following two conditions are satisfied:

(i) the set $\mathcal{C}$ has $(3 g-5)(g-1)$ elements;

(ii) the set $\left\{f_{2,1}\left(\left(x_{a} \wedge x_{b}\right) \otimes x_{c}\right)=x_{b} \otimes x_{a} x_{c}-x_{a} \otimes x_{b} x_{c}\right\}_{(a, b, c) \in S}$ spans ker $f_{1,2}$.

From now on, we assume that $C=R$ is the balanced ribbon of genus $g=2 k+1$ and that $\left\{x_{0}, \ldots, x_{2 k}\right\}$ is the basis of $\mathrm{H}^{0}(\omega)$ described in Proposition 3.1. Our goal for the rest of this section is to construct three monomial bases of cosyzygies for $R$ that will be used in Section 5 to establish the semi-stability of $\operatorname{CoSyz}_{1}(R)$.

Notation. The following terminology will be in force throughout the rest of the paper. We define the $u$-degree of a cosyzygy $\left(x_{a} \wedge x_{b}\right) \otimes x_{c} \in \Gamma$ to be $a+b+c$ and define the level of a tensor $x_{a} \otimes x_{b} x_{c} \in \mathrm{H}^{0}(\omega) \otimes \mathrm{H}^{0}\left(\omega^{2}\right)$ to be $a$. To lighten the notation, we often use $\left(x_{a} \wedge x_{b}\right) \otimes x_{c}$ to denote $f_{2,1}\left(\left(x_{a} \wedge x_{b}\right) \otimes x_{c}\right)=x_{b} \otimes x_{a} x_{c}-x_{a} \otimes x_{b} x_{c}$ in $\mathrm{H}^{0}(\omega) \otimes \mathrm{H}^{0}\left(\omega^{2}\right)$.

For $\alpha \in \mathbb{Q}$, set $\{\alpha\}=\left\lfloor\alpha+\frac{1}{2}\right\rfloor$. In other words, $\{\alpha\}$ is the integer closest to $\alpha$. Observe that for $n \in \mathbb{Z}$, we have

$$
n=\lfloor n / 3\rfloor+\{n / 3\}+\lceil n / 3\rceil .
$$

We use $\langle S\rangle$ to denote the linear span of elements in a subset $S$ of a vector space.

Outline of the construction. We now describe our strategy for constructing monomial bases of cosyzygies for the balanced ribbon $R$. From Definition 4.1, a set $\mathcal{C}=\left\{\left(x_{a} \wedge x_{b}\right) \otimes x_{c}\right\}_{(a, b, c) \in S} \subset$ $\Gamma$ of $(3 g-5)(g-1)$ cosyzygies is a monomial basis of cosyzygies if and only if the images $f_{2,1}\left(\left(x_{a} \wedge x_{b}\right) \otimes x_{c}\right)$ for $(a, b, c) \in S$ span $\operatorname{ker} f_{1,2}$.

The first step in our construction is to write down a suitably chosen set $\mathcal{C}$ of $(3 g-5)(g-1)$ cosyzygies. Next, we make the following observation. Since im $f_{2,1} \subseteq \operatorname{ker} f_{1,2}$ and $f_{1,2}$ is surjective onto $\mathrm{H}^{0}\left(\omega^{3}\right)$, to prove that the images of the cosyzygies in $\mathcal{C}$ span ker $f_{1,2}$, it suffices to show

$$
\operatorname{dim}\left(\mathrm{H}^{0}(\omega) \otimes \mathrm{H}^{0}\left(\omega^{2}\right)\right) /\left\langle f_{2,1}\left(\left(x_{a} \wedge x_{b}\right) \otimes x_{c}\right)\right\rangle_{(a, b, c) \in S} \leqslant \operatorname{dim} \mathrm{H}^{0}\left(\omega^{3}\right)=5(g-1) .
$$

In order to do this, we treat

$$
f_{2,1}\left(\left(x_{a} \wedge x_{b}\right) \otimes x_{c}\right)=x_{b} \otimes x_{a} x_{c}-x_{a} \otimes x_{b} x_{c}
$$

as a relation among the elements of $\mathrm{H}^{0}(\omega) \otimes \mathrm{H}^{0}\left(\omega^{2}\right)$. We therefore reduce to showing that the relations imposed by $\mathcal{C}$ reduce the dimension of $\mathrm{H}^{0}(\omega) \otimes \mathrm{H}^{0}\left(\omega^{2}\right)$ to at most $5(g-1)$. 


\section{TOWARD GIT STABILITY OF SYZYGIES OF CANONICAL CURVES}

The final observation is that all of our results and constructions respect the $\mathbb{G}_{m}$-action on $R$ described in equation (3.2). In particular, we can run our argument by $u$-degree. This observation greatly simplifies our task because the relevant weight spaces have small dimensions. In particular, by Proposition 3.5, we have

$$
\operatorname{dim} \mathrm{H}^{0}\left(\omega^{3}\right)_{d}= \begin{cases}1 & \text { if } 0 \leqslant d \leqslant k \text { or } 5 k \leqslant d \leqslant 6 k \\ 2 & \text { if } k<d<5 k\end{cases}
$$

\subsection{A construction of the first monomial basis}

We define $\mathcal{C}^{+}$to be the union of the following sets of cosyzygies:

(S1) $\left(x_{0} \wedge x_{i}\right) \otimes x_{j}$, where $i \neq 0,2 k$ and $j \neq 2 k$;

(S2) $\left(x_{0} \wedge x_{i}\right) \otimes x_{2 k}$, where $1 \leqslant i \leqslant k-1$;

(S3) $\left(x_{0} \wedge x_{2 k}\right) \otimes x_{i}$, where $i \leqslant k-1$;

(S4) $\left(x_{2 k} \wedge x_{i}\right) \otimes x_{j}$, where $i \neq 0,2 k$ and $j \neq 0$;

(S5) $\left(x_{2 k} \wedge x_{0}\right) \otimes x_{i}$, where $i \geqslant k+1$;

(S6) $\left(x_{2 k} \wedge x_{i}\right) \otimes x_{0}$, where $k+1 \leqslant i \leqslant 2 k-1$;

(S7) $\left(x_{k} \wedge x_{i}\right) \otimes x_{j}$, where $i \neq 0, k, 2 k$ and $j \neq 0,2 k$;

(S8) $\left(x_{k} \wedge x_{0}\right) \otimes x_{2 k}$ and $\left(x_{k} \wedge x_{2 k}\right) \otimes x_{0}$;

(S9) $\left(x_{i} \wedge x_{k+i}\right) \otimes x_{k-i}$, where $1 \leqslant i \leqslant k-1$;

(S10) $\left(x_{2 k-i} \wedge x_{k-i}\right) \otimes x_{k+i}$, where $1 \leqslant i \leqslant k-1$.

Proposition 4.3. The set $\mathcal{C}^{+}$is a monomial basis of cosyzygies for $R$ with $T$-state

$$
w_{T}\left(\mathcal{C}^{+}\right)=\left(g^{2}-1\right)\left(x_{0}+x_{k}+x_{2 k}\right)+(6 g-6) \sum_{i \neq 0, k, 2 k} x_{i}
$$

Proof. Notice that $\mathcal{C}^{+}$contains precisely $(3 g-5)(g-1)$ cosyzygies and that it is invariant under the $\mathbb{Z}_{2}$-involution of the ribbon described in Remark 3.2.

To calculate the $T$-state of $\mathcal{C}^{+}$, observe that $x_{0}, x_{k}, x_{2 k}$ each appear $g^{2}-1$ times, and $x_{i}$, for every $i \neq 0, k, 2 k$, appears $6 g-6$ times. It follows that

$$
w_{T}\left(\mathcal{C}^{+}\right)=\left(g^{2}-1\right)\left(x_{0}+x_{k}+x_{2 k}\right)+(6 g-6) \sum_{i \neq 0, k, 2 k} x_{i} .
$$

We now verify that $\mathcal{C}^{+}$is a monomial basis of cosyzygies. In view of the $\mathbb{Z}_{2}$-symmetry and the dimensions of $\mathrm{H}^{0}\left(\omega^{3}\right)_{d}$ given in (4.2), we need to verify that the quotient $\left(\mathrm{H}^{0}(\omega) \otimes \mathrm{H}^{0}\left(\omega^{2}\right)\right)_{d} /\left\langle\mathcal{C}^{+}\right\rangle_{d}$ is at most 1 -dimensional in $u$-degrees $0 \leqslant d \leqslant k$, and at most 2-dimensional in $u$-degrees $k<$ $d \leqslant 3 k$.

The key player in our argument is the monomial basis $\mathcal{B}^{+}$from Proposition 3.7:

$$
\mathcal{B}^{+}=\left\{x_{0} x_{i}\right\}_{i=0}^{2 k} \cup\left\{x_{k} x_{i}\right\}_{i=1}^{2 k-1} \cup\left\{x_{2 k} x_{i}\right\}_{i=1}^{2 k} .
$$

Tensoring $\mathcal{B}^{+}$with the standard basis $\left\{x_{0}, \ldots, x_{2 k}\right\}$ of $\mathrm{H}^{0}(\omega)$, we obtain the following basis of $\mathrm{H}^{0}(\omega) \otimes \mathrm{H}^{0}\left(\omega^{2}\right)$ :

$$
\mathcal{B}:=\left\{x_{a} \otimes \mathfrak{m}: 0 \leqslant a \leqslant 2 k, \mathfrak{m} \in \mathcal{B}^{+}\right\} .
$$

Our argument now proceeds by $u$-degree. 


\section{A. Deopurkar, M. Fedorchuk and D. Swinarski}

Degree $0 \leqslant d \leqslant k$. We have $\langle\mathcal{B}\rangle_{d}=\left\langle x_{a} \otimes x_{0} x_{d-a}: 0 \leqslant a \leqslant d\right\rangle$. Evidently, we have $x_{a} x_{d-a}=$ $x_{0} x_{d}$ in $\mathrm{H}^{0}\left(\omega^{2}\right)$. It follows that

$$
x_{a} \otimes x_{0} x_{d-a}=x_{0} \otimes x_{a} x_{d-a}+\left(x_{0} \wedge x_{a}\right) \otimes x_{d-a}=x_{0} \otimes x_{0} x_{d}+\left(x_{0} \wedge x_{a}\right) \otimes x_{d-a},
$$

where $\left(x_{0} \wedge x_{a}\right) \otimes x_{d-a}$ is a cosyzygy $(\mathrm{S} 1)$. We conclude that $\langle\mathcal{B}\rangle_{d} /\left\langle\mathcal{C}^{+}\right\rangle_{d}$ is spanned by $x_{0} \otimes x_{0} x_{d}$, hence is at most 1-dimensional.

Degree $k+1 \leqslant d \leqslant 2 k$. We have

$$
\langle\mathcal{B}\rangle_{d}=\left\langle x_{a} \otimes x_{0} x_{d-a}, x_{b} \otimes x_{k} x_{d-k-b}: 0 \leqslant a \leqslant d, 0 \leqslant b<d-k\right\rangle .
$$

If $b \geqslant 1$, using the cosyzygies (S7) and (S1) and Lemma 3.8, we obtain

$$
\begin{aligned}
x_{b} \otimes x_{k} x_{d-k-b} & =x_{k} \otimes x_{b} x_{d-k-b}+\left(x_{k} \wedge x_{b}\right) \otimes x_{d-k-b} \\
& =x_{k} \otimes x_{0} x_{d-k}+\left(x_{k} \wedge x_{b}\right) \otimes x_{d-k-b} \\
& =x_{0} \otimes x_{k} x_{d-k}+\left(x_{0} \wedge x_{k}\right) \otimes x_{d-k}+\left(x_{k} \wedge x_{b}\right) \otimes x_{d-k-b} .
\end{aligned}
$$

Using (S1), we also have

$$
x_{a} \otimes x_{0} x_{d-a}=x_{0} \otimes x_{a} x_{d-a}+\left(x_{0} \wedge x_{a}\right) \otimes x_{d-a} .
$$

It follows that $\langle\mathcal{B}\rangle_{d} /\left\langle\mathcal{C}^{+}\right\rangle_{d}=\left\langle x_{0} \otimes x_{a} x_{d-a}: 0 \leqslant a \leqslant d\right\rangle /\left\langle\mathcal{C}^{+}\right\rangle_{d}$. In other words, every tensor of $u$ degree $d$ is reduced to a tensor of level 0 . Since $\operatorname{dim}\left\langle x_{0} \otimes x_{a} x_{d-a}: 0 \leqslant a \leqslant d\right\rangle=\operatorname{dim} \mathrm{H}^{0}\left(\omega^{2}\right)_{d}=2$, we are done.

Degree $2 k+1 \leqslant d \leqslant 3 k-1$. Write $d=2 k+i$ for $1 \leqslant i \leqslant k-1$. It is easy to see that modulo $\mathcal{C}^{+}$, every tensor in $\left(\mathrm{H}^{0}(\omega) \otimes \mathrm{H}^{0}\left(\omega^{2}\right)\right)_{d}$ can be reduced to a tensor of level 0 , $k$, or $2 k$, by using the cosyzygies $(\mathrm{S} 1)-(\mathrm{S} 4)$ or $(\mathrm{S} 7)$. In other words,

$$
\langle\mathcal{B}\rangle_{d} /\left\langle\mathcal{C}^{+}\right\rangle_{d}=\left\langle x_{0} \otimes x_{k} x_{k+i}, x_{0} \otimes x_{2 k} x_{i}, x_{k} \otimes x_{0} x_{k+i}, x_{k} \otimes x_{k} x_{i}, x_{2 k} \otimes x_{0} x_{i}\right\rangle /\left\langle\mathcal{C}^{+}\right\rangle_{d} .
$$

Since $\operatorname{dim}\left\langle x_{i} \otimes x_{a} x_{2 k-a}: 0 \leqslant a \leqslant 2 k\right\rangle=\operatorname{dim} \mathrm{H}^{0}\left(\omega^{2}\right)_{2 k}=2$, it suffices to show that every tensor in the previous display can be rewritten modulo $\mathcal{C}^{+}$as a tensor of level $i$. First, we observe that

$$
\begin{array}{rlrl}
x_{2 k} \otimes x_{0} x_{i} & =x_{0} \otimes x_{2 k} x_{i}+\left(x_{0} \wedge x_{2 k}\right) \otimes x_{i} & & \text { (using cosyzygy (S3)) } \\
x_{0} \otimes x_{i} x_{2 k}=x_{i} \otimes x_{0} x_{2 k}-\left(x_{0} \wedge x_{i}\right) \otimes x_{2 k} & & \text { (using cosyzygy (S2)) }, \\
x_{k} \otimes x_{i} x_{k}=x_{i} \otimes x_{k}^{2}-\left(x_{k} \wedge x_{i}\right) \otimes x_{k} & & \text { (using cosyzygy (S7)). }
\end{array}
$$

Since $x_{k} \otimes x_{0} x_{k+i}=x_{0} \otimes x_{k} x_{k+i}+\left(x_{0} \wedge x_{k}\right) \otimes x_{k+i}$, it remains to show that $x_{0} \otimes x_{k} x_{k+i}$ can be rewritten as a tensor of level $i$. To this end, we compute

$$
\begin{aligned}
x_{0} \otimes x_{k} x_{k+i} & =x_{k+i} \otimes x_{0} x_{k}-\left(x_{0} \wedge x_{k+i}\right) \otimes x_{k}=x_{k+i} \otimes x_{i} x_{k-i}-\left(x_{0} \wedge x_{k+i}\right) \otimes x_{k} \\
& =x_{i} \otimes x_{k+i} x_{k-i}+\left(x_{i} \wedge x_{k+i}\right) \otimes x_{k-i}-\left(x_{0} \wedge x_{k+i}\right) \otimes x_{k},
\end{aligned}
$$

where we have used a cosyzygy (S9) in the second line.

Degree $d=3 k$. Using the cosyzygies $(\mathbf{S} 1),(\mathbf{S} 4)$, and $(\mathbf{S} 7)$, every tensor in $\langle\mathcal{B}\rangle_{3 k}$ reduces to a tensor of level $0, k$, or $2 k$. It follows that

$$
\langle\mathcal{B}\rangle_{3 k} /\left\langle\mathcal{C}^{+}\right\rangle_{3 k}=\left\langle x_{0} \otimes x_{k} x_{2 k}, x_{k} \otimes x_{0} x_{2 k}, x_{k} \otimes x_{k}^{2}, x_{2 k} \otimes x_{0} x_{k}\right\rangle /\left\langle\mathcal{C}^{+}\right\rangle_{3 k}
$$

Using the cosyzygies (S8), we see that $x_{0} \otimes x_{k} x_{2 k}=x_{k} \otimes x_{0} x_{2 k}$ and $x_{2 k} \otimes x_{0} x_{k}=x_{k} \otimes x_{0} x_{2 k}$ modulo $\mathcal{C}^{+}$. It follows that $\langle\mathcal{B}\rangle_{3 k} /\left\langle\mathcal{C}^{+}\right\rangle_{3 k}$ is spanned by tensors of level $k$, hence is at most 2-dimensional. 


\section{TOWARD GIT STABILITy OF SYZYGIES OF CANONICAL CURVES}

\subsection{A construction of the second monomial basis}

We define $\mathcal{C}^{-}$to be the union of the following sets of cosyzygies:

(T1) $\left(x_{i} \wedge x_{j}\right) \otimes x_{j}$, where $i \notin\{j-k-1, j-k, j, j+k, j+k+1\}$;

(T2) $\left(x_{i} \wedge x_{j+1}\right) \otimes x_{j}$, where $i>j+1$ or $i=j-k+1$, but $i \neq j+k$ and $i \neq j+k+1$;

(T3) $\left(x_{i} \wedge x_{j-1}\right) \otimes x_{j}$, where $i<j-1$ or $i=j+k-1$, but $i \neq j-k$ and $i \neq j-k-1$;

(T4) $\left(x_{i} \wedge x_{j}\right) \otimes x_{j+k}$, where $0<j<k$ and $i \geqslant k$;

(T5) $\left(x_{i} \wedge x_{j}\right) \otimes x_{j+k+1}$, where $0 \leqslant j<k$ and $i \geqslant k$;

(T6) $\left(x_{i} \wedge x_{j+k}\right) \otimes x_{j}$, where $0<j<k$ and $i<k$;

(T7) $\left(x_{i} \wedge x_{j+k+1}\right) \otimes x_{j}$, where $0 \leqslant j<k$ and $i<k$;

(T8) $\left(x_{k} \wedge x_{0}\right) \otimes x_{0}$

(T9) $\left(x_{k} \wedge x_{2 k}\right) \otimes x_{2 k}$;

(T10) $\left(x_{\lfloor(d-2 k) / 3\rfloor} \wedge x_{\lceil(d+2 k) / 3\rceil}\right) \otimes x_{d-\lfloor(d-2 k) / 3\rfloor-\lceil(d+2 k) / 3\rceil}$, where $2 k \leqslant d \leqslant 4 k$, with the following exception: if $k \equiv 1(\bmod 3)$ and $d=2 k$, then take instead $\left(x_{0} \wedge x_{\lfloor 4 k / 3\rfloor}\right) \otimes x_{\lceil 2 k / 3\rceil}$.

The construction of $\mathcal{C}^{-}$is motivated by the following basis of $\mathrm{H}^{0}\left(\omega^{2}\right)$ from Proposition 3.7:

$$
\mathcal{B}^{-}=\left\{x_{i}^{2}\right\}_{i=0}^{2 k} \cup\left\{x_{i} x_{i+1}\right\}_{i=0}^{2 k-1} \cup\left\{x_{i} x_{i+k}\right\}_{i=1}^{k-1} \cup\left\{x_{i} x_{i+k+1}\right\}_{i=0}^{k-1} .
$$

After tensoring with $\left\{x_{0}, \ldots, x_{2 k}\right\}$, the basis above yields the basis of $\mathrm{H}^{0}(\omega) \otimes \mathrm{H}^{0}\left(\omega^{2}\right)$ given by

$$
\mathcal{B}:=\left\{x_{i} \otimes \mathfrak{m} \mid 0 \leqslant i \leqslant 2 k, \mathfrak{m} \in \mathcal{B}^{-}\right\} .
$$

Proposition 4.4. Suppose $k \geqslant 5$, so that $g \geqslant 11$. Then $\mathcal{C}^{-}$is a monomial basis of cosyzygies for $R$ with $T$-state

$$
w_{T}\left(\mathcal{C}^{-}\right)=(7 g-12)\left(x_{0}+x_{2 k}\right)+(7 g-15) x_{k}+(9 g-18) \sum_{i \neq 0, k, 2 k} x_{i} .
$$

Remark 4.5. The exception for $k \equiv 1(\bmod 3)$ and $d=2 k$ in $(\mathbf{T} 10)$ is only necessary to get the correct $T$-state for $\mathcal{C}^{-}$. One obtains a monomial basis regardless.

Proof of Proposition 4.4. Counting cosyzygies of each type in $\mathcal{C}^{-}$, we get $12 k^{2}-4 k=(3 g-$ $5)(g-1)$ cosyzygies. The state calculation is also straightforward. We proceed to verify that $\mathcal{C}^{-}$ is a monomial basis of cosyzygies.

Before moving onto the key technical results used in the proof of Proposition 4.4, we introduce some additional terminology. We call the forms $x_{j}^{2}$ and $x_{j} x_{j+1}$ balanced and the forms $x_{j} x_{j+k}$ and $x_{j} x_{j+k+1} k$-balanced. Likewise, we call a tensor $x_{i} \otimes \mathfrak{m}$ balanced (respectively, $k$-balanced) if $\mathfrak{m}$ is balanced (respectively, $k$-balanced). Finally, we call a balanced tensor $x_{i} \otimes \mathfrak{m}$ of degree $d$ well balanced if $\lfloor(d-2 k) / 3\rfloor \leqslant i \leqslant\lceil(d+2 k) / 3\rceil$. Equivalently, a balanced tensor $x_{i} \otimes x_{s} x_{\ell}$ is well balanced if $\max (|i-s|,|i-\ell|) \leqslant k+1$.

Let $\Lambda$ be the span in $\mathrm{H}^{0}(\omega) \otimes \mathrm{H}^{0}\left(\omega^{2}\right)$ of all cosyzygies in $\mathcal{C}^{-}$, and let $\Lambda^{\prime}$ be the span in $\mathrm{H}^{0}(\omega) \otimes \mathrm{H}^{0}\left(\omega^{2}\right)$ of the cosyzygies (T1)-(T7). Note that the relations given by $\Lambda$ reduce a tensor in $\mathcal{B}$ to a different tensor. For example, modulo (T1) we have

$$
x_{i} \otimes x_{j}^{2}=x_{j} \otimes x_{i} x_{j} .
$$

Our goal is to show that the quotient $\left(\mathrm{H}^{0}(\omega) \otimes \mathrm{H}^{0}\left(\omega^{2}\right)\right) / \Lambda$ is generated by at most one element in degrees $0 \leqslant d \leqslant k$ and $5 k \leqslant d \leqslant 6 k$, and by at most two elements in degrees $k<d<5 k$. Proposition 4.6 does most of the heavy lifting toward this goal and, for the sake of the argument, we assume its statement for now. 


\section{A. Deopurkar, M. Fedorchuk and D. Swinarski}

By Proposition 4.6 and Remark 4.7, the quotient $\left(\mathrm{H}^{0}(\omega) \otimes \mathrm{H}^{0}\left(\omega^{2}\right)\right) / \Lambda^{\prime}$ is generated by one element in degrees $0 \leqslant d<k$ and $5 k<d \leqslant 6 k$, by two elements in degrees $k \leqslant d<2 k$ and $4 k<d \leqslant 5 k$ and by three elements in degrees $2 k \leqslant d \leqslant 4 k$. Therefore, to complete the proof of Proposition 4.4, it suffices to prove that the cosyzygies (T8) and (T9) impose a non-trivial linear relation on the two generators in degree $k$ and $5 k$, respectively, and that the cosyzygy (T10) imposes a non-trivial linear relation on the three generators in degrees $2 k \leqslant d \leqslant 4 k$.

Let $d=k$. The two generators of $\left(\mathrm{H}^{0}(\omega) \otimes \mathrm{H}^{0}\left(\omega^{2}\right)\right) / \Lambda^{\prime}$ in this degree are

$$
\begin{aligned}
\sigma_{1} & :=x_{\{k / 3\}} \otimes x_{\lfloor k / 3\rfloor} x_{\lceil k / 3\rceil} \quad \text { and } \\
\sigma_{2} & :=x_{k} \otimes x_{0}^{2} .
\end{aligned}
$$

The relation imposed by (T8) is

$$
x_{k} \otimes x_{0}^{2}=x_{0} \otimes x_{0} x_{k} .
$$

It is easy to see that by repeatedly applying (T1), (T2), or (T3), and Lemma 3.8, we obtain

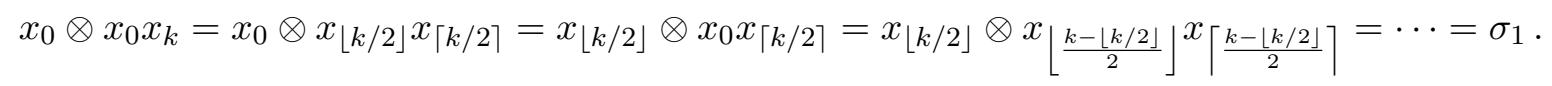

Therefore, (T8) does impose a non-trivial relation $\sigma_{2}=\sigma_{1}$.

The case $d=5 k$ follows symmetrically.

Let $2 k \leqslant d \leqslant 4 k$. The three generators of $\left(\mathrm{H}^{0}(\omega) \otimes \mathrm{H}^{0}\left(\omega^{2}\right)\right) / \Lambda^{\prime}$ in degree $d$ are

$$
\begin{aligned}
& \sigma_{1}:=x_{\{d / 3\}} \otimes x_{\lceil d / 3\rceil} x_{\lfloor d / 3\rfloor}, \\
& \sigma_{2}:=x_{\lceil(d+2 k) / 3\rceil} \otimes x_{\lfloor(d-k) / 3\rfloor} x_{\{(d-k) / 3\}}, \quad \text { and } \\
& \sigma_{3}:=x_{\lfloor(d-2 k) / 3\rfloor} \otimes x_{\{(d+k) / 3\}} x_{\lceil(d+k) / 3\rceil} .
\end{aligned}
$$

For brevity, set $\ell=\lceil(d+2 k) / 3\rceil$ and $s=\lfloor(d-2 k) / 3\rfloor$. The relation imposed by (T10) is

$$
x_{\ell} \otimes x_{s} x_{d-\ell-s}=x_{s} \otimes x_{\ell} x_{d-\ell-s} .
$$

Assume $d \leqslant 3 k$; the case $d \geqslant 3 k$ follows symmetrically. Since $d \leqslant 3 k$, we have

$$
s<\lfloor(d-k) / 3\rfloor \leqslant\{(d-k) / 3\}<d-\ell-s \leqslant k .
$$

On the left-hand side of (4.4), we have by Lemma 3.8

$$
\begin{aligned}
x_{\ell} \otimes x_{s} x_{d-\ell-s} & =x_{\ell} \otimes x_{\lfloor(d-k) / 3\rfloor} x_{\{(d-k) / 3\}} \\
& =\sigma_{2} .
\end{aligned}
$$

On the right-hand side of (4.4), working modulo (T6) and (T7), and applying Lemma 3.8, we get

$$
\begin{aligned}
x_{s} \otimes x_{\ell} x_{d-\ell-s} & =\lambda x_{s} \otimes x_{\{(d+k) / 3\}} x_{\lceil(d+k) / 3\rceil}+\mu x_{s} \otimes x_{\lfloor(d-s-k) / 2\rfloor} x_{\lceil(d-s+k) / 2\rceil}, \\
& =\lambda \sigma_{3}+\mu x_{\lceil(d-s+k) / 2\rceil} \otimes \mathfrak{m}, \quad \text { where } \mathfrak{m} \text { is balanced, } \\
& =\lambda \sigma_{3}+\mu\left(\alpha \sigma_{1}+\beta \sigma_{2}+\gamma \sigma_{3}\right),
\end{aligned}
$$

where the last step uses Proposition 4.6. Furthermore, since $k \geqslant 5$, we have $\lceil(d-s+k) / 2\rceil>$ $\lceil(d+2 k) / 3\rceil$. Hence Proposition 4.6(ii)(c) implies $\alpha<0$. Thus, (T10) imposes a relation

$$
\sigma_{2}=\lambda \sigma_{3}+\mu\left(\alpha \sigma_{1}+\beta \sigma_{2}+\gamma \sigma_{3}\right) .
$$

If $\mu=0$, then this relation is clearly non-trivial. If $\mu \neq 0$, the non-vanishing of the coefficient of $\sigma_{1}$ shows that the relation is non-trivial. 


\section{TOWARD GIT STABILITY OF SYZYGIES OF CANONICAL CURVES}

Finally, we verify that the exceptional cosyzygy in (T10) for $k \equiv 1(\bmod 3)$ and $d=2 k$ imposes a non-trivial relation. The argument is almost the same. In this case, the cosyzygy gives

$$
x_{\lfloor 4 k / 3\rfloor} \otimes x_{0} x_{\lceil 2 k / 3\rceil}=x_{0} \otimes x_{\lceil 2 k / 3\rceil} x_{\lfloor 4 k / 3\rfloor} \cdot
$$

Applying Lemma 3.8 and Proposition 4.6(i), we obtain

$$
\begin{aligned}
x_{\lfloor 4 k / 3\rfloor} \otimes x_{0} x_{\lceil 2 k / 3\rceil} & =x_{\lfloor 4 k / 3\rfloor} \otimes \mathfrak{m}, \quad \text { where } \mathfrak{m} \text { is balanced, } \\
& =\alpha \sigma_{1}+\beta \sigma_{2}+\gamma \sigma_{3} .
\end{aligned}
$$

Since $\lfloor(d-2 k) / 3\rfloor<\lfloor 4 k / 3\rfloor<\lceil(d+2 k) / 3\rceil$, Proposition 4.6(ii)(b) says that $\alpha>0$.

Similarly, reducing the right-hand side of (4.5), we get

$$
\begin{aligned}
x_{0} \otimes x_{\lceil 2 k / 3\rceil} x_{\lfloor 4 k / 3\rfloor} & =\lambda x_{0} \otimes x_{k}^{2}+\mu x_{0} \otimes x_{\lfloor k / 2\rfloor} x_{\lceil 3 k / 2\rceil}, \quad \text { where } \lambda, \mu>0, \\
& =\lambda \sigma_{3}+\mu x_{\lceil 3 k / 2\rceil} \otimes \mathfrak{n}, \quad \text { where } \mathfrak{n} \text { is balanced, } \\
& =\lambda \sigma_{3}+\mu\left(\alpha^{\prime} \sigma_{1}+\beta^{\prime} \sigma_{2}+\gamma^{\prime} \sigma_{3}\right) .
\end{aligned}
$$

Since $\lceil 3 k / 2\rceil>\lceil(d+2 k) / 3\rceil$, Proposition 4.6(ii)(c) implies $\alpha^{\prime}<0$. Thus, (T10) imposes

$$
\alpha \sigma_{1}+\beta \sigma_{2}+\gamma \sigma_{3}=\lambda \sigma_{3}+\mu\left(\alpha^{\prime} \sigma_{1}+\beta^{\prime} \sigma_{2}+\gamma^{\prime} \sigma_{3}\right) .
$$

Since $\alpha>0$ whereas $\mu \alpha^{\prime}<0$, the relation is non-trivial.

Proposition 4.6. (i) Every element of $\left(\mathrm{H}^{0}(\omega) \otimes \mathrm{H}^{0}\left(\omega^{2}\right)\right) / \Lambda^{\prime}$ can be uniquely expressed as a linear combination of the following tensors:

$$
\begin{aligned}
& \text { (type 1) } \begin{cases}x_{i} \otimes x_{i}^{2}, & \text { where } 0 \leqslant i \leqslant 2 k, \\
x_{i} \otimes x_{i} x_{i+1}, & \text { where } 0 \leqslant i \leqslant 2 k-1, \\
x_{i} \otimes x_{i-1} x_{i}, & \text { where } 1 \leqslant i \leqslant 2 k,\end{cases} \\
& \text { (type } 2) \begin{cases}x_{i+k} \otimes x_{i}^{2}, & \text { where } 0 \leqslant i \leqslant k, \\
x_{i+k+1} \otimes x_{i}^{2}, & \text { where } 0 \leqslant i \leqslant k-1, \\
x_{i+k+1} \otimes x_{i} x_{i+1}, & \text { where } 0 \leqslant i \leqslant k-1,\end{cases} \\
& \text { (type } 3) \begin{cases}x_{i-k} \otimes x_{i}^{2}, & \text { where } k \leqslant i \leqslant 2 k, \\
x_{i-k-1} \otimes x_{i}^{2}, & \text { where } k+1 \leqslant i \leqslant 2 k, \\
x_{i-k-1} \otimes x_{i} x_{i-1}, & \text { where } k+1 \leqslant i \leqslant 2 k .\end{cases}
\end{aligned}
$$

(ii) Furthermore, let $2 k \leqslant d \leqslant 4 k$. Then there is precisely one tensor of degree $d$ of each type 1-3. Suppose that the balanced tensor $\tau=x_{i} \otimes x_{\lfloor(d-i) / 2\rfloor} x_{\lceil(d-i) / 2\rceil}$ is expressed as

$$
\tau=\alpha \sigma_{1}+\beta \sigma_{2}+\gamma \sigma_{3},
$$

where $\sigma_{t}$ is of type $t$. Then

(a) $\alpha+\beta+\gamma=1$;

(b) if $\lfloor(d-2 k) / 3\rfloor<i<\lceil(d+2 k) / 3\rceil$, then $\alpha>0, \beta \geqslant 0, \gamma \geqslant 0$ (well-balanced case);

(c) if $i>\lceil(d+2 k) / 3\rceil$, then $\alpha<0, \beta>1, \gamma \leqslant 0$;

(d) if $i<\lfloor(d-2 k) / 3\rfloor$, then $\alpha<0, \beta \leqslant 0, \gamma>1$.

Remark 4.7. In terms of the $u$-degree $d$, the list of tensors in Proposition 4.6 can be written more compactly as follows:

(type 1) $\quad x_{\{d / 3\}} \otimes x_{\lfloor d / 3\rfloor} x_{\lceil d / 3\rceil}$, where $0 \leqslant d \leqslant 6 k ;$ 


\section{A. Deopurkar, M. Fedorchuk and D. Swinarski}

(type 2) $\quad x_{\lceil(d+2 k) / 3\rceil} \otimes x_{\lfloor(d-k) / 3\rfloor} x_{\{(d-k) / 3\}}$, where $k \leqslant d \leqslant 4 k$;

(type 3) $\quad x_{\lfloor(d-2 k) / 3\rfloor} \otimes x_{\lceil(d+k) / 3\rceil} x_{\{(d+k) / 3\}}$, where $2 k \leqslant d \leqslant 5 k$.

Proof of Proposition 4.6. Using the cosyzygies (T1)-(T7), we reduce every element of the basis $\mathcal{B}$ to a linear combination of the tensors of type 1,2 , and 3 . Uniqueness then follows by counting the dimensions.

Step 1: Reducing $k$-balanced tensors to balanced tensors. Consider a $k$-balanced tensor $x_{i} \otimes x_{\lfloor(d-i-k) / 2\rfloor} x_{\lceil(d-i+k) / 2\rceil}$, where $k \leqslant d-i \leqslant 3 k$. Suppose $i \geqslant k$. Then modulo the cosyzygy (T4) or (T5), we get

$$
x_{i} \otimes x_{\lfloor(d-i-k) / 2\rfloor} x_{\lceil(d-i+k) / 2\rceil}=x_{\lfloor(d-i-k) / 2\rfloor} \otimes x_{i} x_{\lceil(d-i+k) / 2\rceil} \cdot
$$

Since $i \geqslant k$ and $\lceil(d-i+k) / 2\rceil \geqslant k$, the form $x_{i} x_{\lceil(d-i+k) / 2\rceil}$ is equal to a balanced form in $\mathrm{H}^{0}\left(\omega^{2}\right)$ by Lemma 3.8. The case $i<k$ is analogous using cosyzygies (T6) or (T7).

Step 2: Reducing balanced tensors to well-balanced tensors. Consider a balanced tensor $x_{i} \otimes x_{\lfloor(d-i) / 2\rfloor} x_{\lceil(d-i) / 2\rceil}$ that is not well balanced. For brevity, set

$$
s=\lfloor(d-i) / 2\rfloor, \quad \ell=\lceil(d-i) / 2\rceil .
$$

Assume $i>\lceil(d+2 k) / 3\rceil$ (the case $i<\lfloor(d-2 k) / 3\rfloor$ follows symmetrically). We then have $i-s>k+1$ and hence $i>\ell+k>\ell$. Modulo the cosyzygy (T1) or (T2), we get

$$
x_{i} \otimes x_{s} x_{\ell}=x_{\ell} \otimes x_{s} x_{i} .
$$

By Lemma 3.8, we have

$$
x_{s} x_{i}=\lambda \mathfrak{m}_{1}+\mu \mathfrak{m}_{1}^{\prime},
$$

where $\mathfrak{m}_{1}$ is balanced, $\mathfrak{m}_{1}^{\prime}$ is $k$-balanced, and $\lambda+\mu=1$. Since $i-s>k+1$, we also have $\lambda<0$. Reducing the $k$-balanced tensor $x_{\ell} \otimes \mathfrak{m}_{1}^{\prime}$ as in Step 1, we get

$$
\begin{aligned}
x_{\ell} \otimes \mathfrak{m}_{1}^{\prime} & =x_{\ell} \otimes x_{\lfloor(d-\ell-k) / 2\rfloor} x_{\lceil(d-\ell+k) / 2\rceil} \\
& =x_{\lceil(d-\ell+k) / 2\rceil} \otimes \mathfrak{m}_{2} \quad \text { modulo (T6) or (T7), }
\end{aligned}
$$

where $\mathfrak{m}_{2}$ is balanced. We thus get an expression

$$
x_{i} \otimes x_{s} x_{\ell}=\lambda x_{\ell} \otimes \mathfrak{m}_{1}+\mu x_{\lceil(d-\ell+k) / 2\rceil} \otimes \mathfrak{m}_{2},
$$

where $\mathfrak{m}_{1}$ and $\mathfrak{m}_{2}$ are balanced, $s=\lfloor(d-i) / 2\rfloor, \ell=\lceil(d-i) / 2\rceil, \lambda+\mu=1, \lambda<0$.

Note that we have the inequalities

$$
\begin{aligned}
& \lfloor(d-2 k) / 3\rfloor \leqslant \ell \leqslant\lceil(d+2 k) / 3\rceil \text { and } \\
& \lceil(d+2 k) / 3\rceil \leqslant\lceil(d-\ell+k) / 2\rceil<i .
\end{aligned}
$$

In other words, the first tensor on the right in (4.6) is already well balanced and the second is strictly closer to being well balanced than the original tensor. By repeated application of (4.6), we arrive at a linear combination of well-balanced tensors.

Step 3: Reducing well-balanced tensors. We now show that all well-balanced tensors reduce to linear combinations of tensors of type 1,2, and 3 . We will make use of the following result.

Lemma 4.8. Let $\tau=x_{i} \otimes x_{\lfloor(d-i) / 2\rfloor} x_{\lceil(d-i) / 2\rceil}$ be a well-balanced tensor of degree $d$. Modulo (T1)-(T7), we have a reduction

$$
\tau=\lambda \tau_{1}+\mu \tau_{2}
$$




\section{TOWARD GIT STABILITY OF SYZYGIES OF CANONICAL CURVES}

where $\tau_{1}$ and $\tau_{2}$ are well balanced, $\lambda+\mu=1$, and $\lambda, \mu \geqslant 0$. Moreover, if $\tau$ is not of type 2 or 3 , then $\lambda>0$. And, if $\tau$ is not of type 1 , 2 , or 3 , then $\tau_{1}=x_{j} \otimes x_{\lfloor(d-j) / 2\rfloor} x_{\lceil(d-j) / 2\rceil}$, where $|\{d / 3\}-j|<|\{d / 3\}-i|$, and $\tau_{1}$ is not of type 2 or 3 .

Proof of Lemma 4.8. Let $\tau=x_{i} \otimes x_{\lfloor(d-i) / 2\rfloor} x_{\lceil(d-i) / 2\rceil}$. For brevity, set $s=\lfloor(d-i) / 2\rfloor$ and $\ell=\lceil(d-i) / 2\rceil$. If $i=\ell$ or $i=s$, then $\tau$ is of type 1 . In this case, we take $\tau_{1}=\tau$ and $\lambda=1$, $\mu=0$. If both $x_{i} x_{\ell}$ and $x_{i} x_{s}$ are $k$-balanced, then $\tau$ is of type 2 or 3 . In this case, we take $\tau_{2}=\tau$ and $\lambda=0, \mu=1$. Suppose neither of these is the case. We consider the case $i>\ell$; the case $i<s$ follows symmetrically. Note that $\ell$ satisfies

$$
\{(d-k) / 3\} \leqslant \ell \leqslant\{(d+k) / 3\} .
$$

We first treat the special case $i=s+k$. Since $x_{i} x_{\ell}$ and $x_{i} x_{s}$ are not both $k$-balanced by our assumption, we must have $s=\ell-1$. Therefore, we get

$$
\begin{aligned}
\tau= & x_{i} \otimes x_{\ell-1} x_{\ell} \\
= & x_{\ell-1} \otimes x_{i} x_{\ell} \quad \text { modulo }(\mathbf{T} 3) \\
= & \lambda x_{\ell-1} \otimes \mathfrak{m}_{1}+\mu x_{\ell-1} \otimes x_{\ell+k} x_{\ell-1}, \\
& \quad \text { where } \mathfrak{m}_{1} \text { is balanced, } \lambda>0, \mu \geqslant 0, \text { and } \lambda+\mu=1 \text { (Lemma 3.8) } \\
= & \lambda x_{\ell-1} \otimes \mathfrak{m}_{1}+\mu x_{\ell+k} \otimes x_{\ell-1} x_{\ell-1} \quad \text { modulo }(\mathbf{T} 6) \\
= & \lambda \tau_{1}+\mu \tau_{2}, \quad \text { as desired. }
\end{aligned}
$$

Now assume $i \neq s+k$. Then $0<i-\ell \leqslant i-s<k$. In this case, we get

$$
\begin{aligned}
\tau & =x_{i} \otimes x_{s} x_{\ell} \\
& =x_{\ell} \otimes x_{s} x_{i} \quad \text { modulo (T1) or (T2). }
\end{aligned}
$$

Using Lemma 3.8, we have

$$
x_{s} x_{i}=\lambda \mathfrak{m}_{1}+\mu \mathfrak{m}_{1}^{\prime},
$$

where $\mathfrak{m}_{1}$ is balanced, $\mathfrak{m}_{1}^{\prime}$ is $k$-balanced, and $\lambda+\mu=1$. Since $0<i-s<k$, we have $\lambda>0$ and $\mu \geqslant 0$. Reducing the $k$-balanced tensor $x_{\ell} \otimes \mathfrak{m}_{1}^{\prime}$ as in Step 1, we get

$$
x_{\ell} \otimes \mathfrak{m}_{1}^{\prime}=x_{p} \otimes \mathfrak{m}_{2},
$$

where $\mathfrak{m}_{2}$ is balanced and

In either case, (4.8) implies

$$
p= \begin{cases}\lfloor(d-\ell-k) / 2\rfloor & \text { if } \ell \geqslant k, \\ \lceil(d-\ell+k) / 2\rceil & \text { if } \ell<k .\end{cases}
$$

$$
\lfloor(d-2 k) / 3\rfloor \leqslant p \leqslant\lceil(d+2 k) / 3\rceil .
$$

Setting $\tau_{1}=x_{\ell} \otimes \mathfrak{m}_{1}$ and $\tau_{2}=x_{p} \otimes \mathfrak{m}_{2}$, we thus get

$$
\tau=\lambda \tau_{1}+\mu \tau_{2},
$$

as claimed.

Finally, we note that if $\tau=x_{i} \otimes x_{s} x_{\ell}$ is not of type 1,2 , or 3 , then by construction $\tau_{1}$ has level $j$ where either $j=s$ in the case $i=s+k$, or $j=\ell$ in all other cases. In either case, it is clear that $|\{d / 3\}-j|<|\{d / 3\}-i|$. (Informally, this means that $\tau_{1}$ is closer to being of type 1 than $\tau$.) This finishes the proof of the lemma. 


\section{A. Deopurkar, M. Fedorchuk and D. Swinarski}

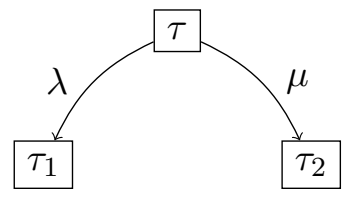

FiguRE 1. The relations among well-balanced tensors as a Markov chain

Proof of Proposition 4.6, continued. Let $\Omega$ be the set of well-balanced tensors. Define a linear operator $P:\langle\Omega\rangle \rightarrow\langle\Omega\rangle$ that encodes (4.7), namely

$$
P: \tau \mapsto \lambda \tau_{1}+\mu \tau_{2} .
$$

By Lemma 4.8, we can interpret $P$ as a Markov process on $\Omega$ (see Figure 1 ). Notice that the absorbing states of this Markov chain are precisely the tensors of type 1, 2, and 3. Furthermore, from every other tensor, the path $\tau \rightarrow \tau_{1} \rightarrow \cdots$ eventually leads to a tensor of type 1 , again by Lemma 4.8. As a result, $P$ is an absorbing Markov chain. By basic theory of Markov chains, for every $v \in\langle\Omega\rangle$, the $\operatorname{limit}_{n \rightarrow \infty} \lim _{n} v$ exists and is supported on the absorbing states. Taking $v=1 \cdot \tau$, we conclude that $\tau$ reduces to a linear combination of the absorbing states. We thus get a linear relation

$$
\tau=\alpha \sigma_{1}+\beta \sigma_{2}+\gamma \sigma_{3}
$$

where $\sigma_{t}$ is of type $t$, as claimed.

The above analysis also lets us deduce the claims about the coefficients from part (ii) of the proposition. Let $2 k \leqslant d \leqslant 4 k$. Say $\tau=x_{i} \otimes x_{s} x_{\ell}$ reduces as

$$
\tau=\alpha \sigma_{1}+\beta \sigma_{2}+\gamma \sigma_{3}
$$

where $\sigma_{t}$ is of type $t$.

For part (ii)(a), we note that $\alpha+\beta+\gamma=1$ follows by passing to $\mathrm{H}^{0}\left(\omega^{3}\right)$ and comparing the coefficients of $u^{d}$.

For part (ii)(b), assume $\lfloor(d-2 k) / 3\rfloor<i<\lceil(d+2 k) / 3\rceil$. Then $\tau$ is well balanced. The non-negativity of $P$ implies the non-negativity of $\alpha, \beta$, and $\gamma$. Furthermore, since there is a path of positive weight from $\tau$ to $\sigma_{1}$, we have $\alpha>0$.

For part (ii)(c), note that if $i=\lceil(d+2 k) / 3\rceil$, then $\alpha=0, \beta=1$, and $\gamma=0$. For $i>$ $\lceil(d+2 k) / 3\rceil$, we show by descending induction on $i$ that $\alpha<0$ and $\gamma \leqslant 0$. Then since $\alpha+\beta+\gamma=1$, it follows that $\beta>1$. For the induction, recall the reduction (4.6)

$$
\tau=\lambda x_{\ell} \otimes \mathfrak{m}_{1}+\mu x_{\lceil(d-\ell+k) / 2\rceil} \otimes \mathfrak{m}_{2},
$$

where the $\mathfrak{m}_{i}$ are balanced, $\lambda<0, \mu>0$, and $\lambda+\mu=1$. Recall also the inequalities

$$
\begin{aligned}
& \lfloor(d-2 k) / 3\rfloor \leqslant \ell \leqslant\lceil(d+2 k) / 3\rceil \text { and } \\
& \lceil(d+2 k) / 3\rceil \leqslant\lceil(d-\ell+k) / 2\rceil<i .
\end{aligned}
$$

Except in the extreme case $(d, i)=(2 k, 2 k)$, both inequalities in the first line are strict. Say we have the reductions

$$
\begin{aligned}
x_{\ell} \otimes \mathfrak{m}_{1} & =\alpha^{\prime} \sigma_{1}+\beta^{\prime} \sigma_{2}+\gamma^{\prime} \sigma_{3} \text { and } \\
x_{\lceil(d-\ell+k) / 2\rceil} \otimes \mathfrak{m}_{2} & =\alpha^{\prime \prime} \sigma_{1}+\beta^{\prime \prime} \sigma_{2}+\gamma^{\prime \prime} \sigma_{3} .
\end{aligned}
$$

By part (ii)(b), we have $\alpha^{\prime}>0$ and $\gamma^{\prime} \geqslant 0$. By the inductive assumption, we have $\alpha^{\prime \prime} \leqslant 0$ and $\gamma^{\prime \prime} \leqslant 0$. Since $\lambda<0$ and $\mu>0$ in (4.6), we conclude the induction step. In the extreme case 


\section{TOWARD GIT STABILITY OF SYZYGIES OF CANONICAL CURVES}

$(d, i)=(2 k, 2 k)$, the reduction $(4.6)$ becomes

$$
\tau=\lambda \sigma_{3}+\mu x_{\lceil 3 k / 2\rceil} \otimes \mathfrak{m}_{2} .
$$

The assertion now follows from that for $x_{\lceil 3 k / 2\rceil} \otimes \mathfrak{m}_{2}$.

Finally, part (ii)(d) follows symmetrically from part (ii)(c).

\subsection{A construction of the third (and final!) monomial basis}

Let $\mathcal{C}^{\star}$ be the union of the following sets of cosyzygies:

(U1) the cosyzygies (T1)-(T9) in the description of $\mathcal{C}^{-}$from Subsection 4.2;

(U2) $\left(x_{d-k} \wedge x_{0}\right) \otimes x_{k}$ for $2 k \leqslant d<3 k$;

(U3) $\left(x_{2 k} \wedge x_{0}\right) \otimes x_{k}$;

(U4) $\left(x_{d-3 k} \wedge x_{2 k}\right) \otimes x_{k}$ for $3 k<d \leqslant 4 k$.

Proposition 4.9. The set $\mathcal{C}^{\star}$ is a monomial basis of cosyzygies for $R$ with $T$-state

$$
w_{T}\left(\mathcal{C}^{\star}\right)=\frac{15 g-29}{2}\left(x_{0}+x_{2 k}\right)+(8 g-16) x_{k}+(9 g-20) \sum_{i \neq 0, k, 2 k} x_{i} .
$$

Proof. Let $\Lambda^{\prime}$ be the span in $\mathrm{H}^{0}(\omega) \otimes \mathrm{H}^{0}\left(\omega^{2}\right)$ of the cosyzygies in (U1). Then by Proposition 4.6 and the proof of Proposition 4.4 for the $u$-degrees $d=k$ and $d=5 k$, the quotient $\left(\mathrm{H}^{0}(\omega) \otimes \mathrm{H}^{0}\left(\omega^{2}\right)\right) / \Lambda^{\prime}$ is generated by one element in degrees $0 \leqslant d \leqslant k$ and $5 k \leqslant d \leqslant 6 k$, by two elements in degrees $k<d<2 k$ and $4 k<d<5 k$, and by three elements in degrees $2 k \leqslant d \leqslant 4 k$. It suffices to prove that the cosyzygies (U2)-(U4) impose a non-trivial linear relation among the three generators in degrees $2 k \leqslant d \leqslant 4 k$.

Let $2 k \leqslant d<3 k$. Recall that by Proposition 4.6(i) the three generators in this degree are

$$
\begin{aligned}
\sigma_{1} & :=x_{\{d / 3\}} \otimes x_{\lceil d / 3\rceil} x_{\lfloor d / 3\rfloor}, \\
\sigma_{2} & :=x_{\lceil(d+2 k) / 3\rceil} \otimes x_{\lfloor(d-k) / 3\rfloor} x_{\{(d-k) / 3\}}, \quad \text { and } \\
\sigma_{3} & :=x_{\lfloor(d-2 k) / 3\rfloor} \otimes x_{\{(d+k) / 3\}} x_{\lceil(d+k) / 3\rceil} .
\end{aligned}
$$

The relation given by (U2) is

$$
x_{0} \otimes x_{d-k} x_{k}=x_{d-k} \otimes x_{0} x_{k} .
$$

We reduce both sides modulo $\Lambda^{\prime}$. Note that $x_{0} \otimes x_{d-k} x_{k}=x_{0} \otimes \mathfrak{m}_{1}$ and $x_{d-k} \otimes x_{0} x_{k}=x_{d-k} \otimes \mathfrak{m}_{2}$, where the $\mathfrak{m}_{i}$ are balanced. Modulo $\Lambda^{\prime}$, we have by Proposition 4.6

$$
\begin{aligned}
x_{0} \otimes \mathfrak{m}_{1} & =\alpha \sigma_{1}+\beta \sigma_{2}+\gamma \sigma_{3} \quad \text { and } \\
x_{d-k} \otimes \mathfrak{m}_{2} & =\alpha^{\prime} \sigma_{1}+\beta^{\prime} \sigma_{2}+\gamma^{\prime} \sigma_{3} .
\end{aligned}
$$

The relation imposed by (U2) is therefore

$$
\alpha \sigma_{1}+\beta \sigma_{2}+\gamma \sigma_{3}=\alpha^{\prime} \sigma_{1}+\beta^{\prime} \sigma_{2}+\gamma^{\prime} \sigma_{3} .
$$

On the one hand, since $0 \leqslant\lfloor(d-2 k) / 3\rfloor$, Proposition 4.6(ii)(d) implies $\gamma>0, \alpha \leqslant 0$, and $\beta \leqslant 0$. On the other hand, since $\lfloor(d-2 k) / 3\rfloor<d-k$, we have either $\alpha^{\prime}>0$ (if $d-k<\lceil(d+2 k) / 3\rceil$ ) or $\beta^{\prime}>0$ (if $\left.\lceil(d+2 k) / 3\rceil \leqslant d-k\right)$. In either case, relation (4.9) is non-trivial.

The same argument goes through for $d=3 k$.

The case $3 k<d \leqslant 4 k$ follows symmetrically. 


\section{A. Deopurkar, M. Fedorchuk and D. Swinarski}

We finish this section with an existence result for monomial bases of cosyzygies in low genus. Recall that the set $\mathcal{C}^{-}$constructed in Section 4.2 is a monomial basis of cosyzygies for $R$ only if $g \geqslant 11$. The following proposition shows how to modify the basis in low genus.

Proposition 4.10. As before, $R$ is the balanced ribbon of genus $g$.

(i) Suppose $g=7$. There exists a monomial basis $\mathcal{C}^{-}$of cosyzygies for $R$ with $T$-state

$$
w_{T}\left(\mathcal{C}^{-}\right)=40 x_{0}+42 x_{1}+42 x_{2}+40 x_{3}+42 x_{4}+42 x_{5}+40 x_{6} .
$$

(ii) Suppose $g=9$. There exists a monomial basis $\mathcal{C}^{-}$of cosyzygies for $R$ with $T$-state

$$
w_{T}\left(\mathcal{C}^{-}\right)=56 x_{0}+60 x_{1}+60 x_{2}+60 x_{3}+56 x_{4}+60 x_{5}+60 x_{6}+60 x_{7}+56 x_{8} .
$$

Proof. Both statements are proved by explicitly writing down a monomial basis of cosyzygies of $R$. We provide details only in the case $g=7$, the case $g=9$ being similar. Let $\mathcal{C}^{-}$be the following union of sets of cosyzygies:

(V1) $u$-degree 1: $x_{0} \wedge x_{1} \otimes x_{0}$

(V2) $u$-degree 2: $x_{0} \wedge x_{2} \otimes x_{0}, x_{0} \wedge x_{1} \otimes x_{1}$;

(V3) $u$-degree 3: $x_{0} \wedge x_{3} \otimes x_{0}, x_{0} \wedge x_{1} \otimes x_{2}, x_{0} \wedge x_{2} \otimes x_{1}$;

(V4) $u$-degree 4: $x_{0} \wedge x_{4} \otimes x_{0}, x_{0} \wedge x_{3} \otimes x_{1}, x_{1} \wedge x_{2} \otimes x_{1}, x_{0} \wedge x_{2} \otimes x_{2}$;

(V5) $u$-degree 5: $x_{0} \wedge x_{5} \otimes x_{0}, x_{0} \wedge x_{4} \otimes x_{1}, x_{1} \wedge x_{3} \otimes x_{1}, x_{1} \wedge x_{2} \otimes x_{2}, x_{0} \wedge x_{2} \otimes x_{3}, x_{0} \wedge x_{1} \otimes x_{4}$;

(V6) $u$-degree 6: $x_{0} \wedge x_{6} \otimes x_{0}, x_{0} \wedge x_{5} \otimes x_{1}, x_{1} \wedge x_{4} \otimes x_{1}, x_{2} \wedge x_{3} \otimes x_{1}, x_{0} \wedge x_{2} \otimes x_{4}, x_{1} \wedge x_{2} \otimes x_{3}$, $x_{0} \wedge x_{3} \otimes x_{3}, x_{0} \wedge x_{1} \otimes x_{5}$

(V7) $u$-degree 7: $x_{0} \wedge x_{6} \otimes x_{1}, x_{1} \wedge x_{5} \otimes x_{1}, x_{1} \wedge x_{4} \otimes x_{2}, x_{2} \wedge x_{3} \otimes x_{2}, x_{0} \wedge x_{3} \otimes x_{4}, x_{1} \wedge x_{2} \otimes x_{4}$, $x_{1} \wedge x_{3} \otimes x_{3}, x_{0} \wedge x_{1} \otimes x_{6}, x_{0} \wedge x_{2} \otimes x_{5}$

(V8) $u$-degree 8: $x_{2} \wedge x_{6} \otimes x_{0}, x_{2} \wedge x_{5} \otimes x_{1}, x_{2} \wedge x_{4} \otimes x_{2}, x_{0} \wedge x_{4} \otimes x_{4}, x_{2} \wedge x_{3} \otimes x_{3}, x_{1} \wedge x_{3} \otimes x_{4}$, $x_{1} \wedge x_{2} \otimes x_{5}, x_{1} \wedge x_{4} \otimes x_{3}, x_{0} \wedge x_{3} \otimes x_{5}, x_{0} \wedge x_{2} \otimes x_{6}$

(V9) $u$-degree 9: $x_{3} \wedge x_{6} \otimes x_{0}, x_{3} \wedge x_{5} \otimes x_{1}, x_{4} \wedge x_{5} \otimes x_{0}, x_{3} \wedge x_{4} \otimes x_{2}, x_{1} \wedge x_{4} \otimes x_{4}, x_{2} \wedge x_{3} \otimes x_{4}$, $x_{2} \wedge x_{5} \otimes x_{2}, x_{1} \wedge x_{3} \otimes x_{5}, x_{1} \wedge x_{2} \otimes x_{6}, x_{0} \wedge x_{3} \otimes x_{6}$

(V10) $u$-degrees 10-18: the cosyzygies that are obtained from those above by the $\mathbb{Z}_{2}$-involution described in Remark 3.2.

A straightforward but tedious linear algebra calculation, which we omit, shows that $\mathcal{C}^{-}$is a monomial basis of cosyzygies for the genus 7 balanced ribbon. The $T$-state of $\mathcal{C}^{-}$is clearly

$$
40 x_{0}+42 x_{1}+42 x_{2}+40 x_{3}+42 x_{4}+42 x_{5}+40 x_{6} .
$$

We note in closing that both parts of the proposition can also be verified by a direct search using a computer.

\section{Semi-stability of the first syzygy point}

In this section, we prove that the balanced canonical ribbon $R$ has semi-stable first syzygy point, thus obtaining our main result. We begin with two preliminary lemmas.

Lemma 5.1. For the balanced canonical ribbon $R$ of odd genus $g \geqslant 5$, we have $K_{1,2}(R)=0$.

Proof. The claim follows from the existence of a single monomial basis of cosyzygies. We exhibited such a basis in Proposition 4.3. 


\section{TOWARD GIT STABILITy OF SYZYGIES OF CANONICAL CURVES}

Remark 5.2. In the terminology of Bayer and Eisenbud [BE95, §2], the Clifford index of the genus $2 k+1$ balanced ribbon $R$ is $k$. Therefore, Lemma 5.1 is an immediate consequence of Green's conjecture for $R$, which is still open to the best of our knowledge.

Recall from Section 4 that we have constructed three monomial bases of cosyzygies for $R$, namely, $\mathcal{C}^{+}, \mathcal{C}^{-}$, and $\mathcal{C}^{\star}$, with the following $T$-states:

$$
\begin{aligned}
& w_{T}\left(\mathcal{C}^{+}\right)=\left(g^{2}-1\right)\left(x_{0}+x_{k}+x_{2 k}\right)+(6 g-6) \sum_{i \neq 0, k, 2 k} x_{i}, \\
& w_{T}\left(\mathcal{C}^{-}\right)=40 x_{0}+42 x_{1}+42 x_{2}+40 x_{3}+42 x_{4}+42 x_{5}+40 x_{6} \quad \text { if } g=7, \\
& w_{T}\left(\mathcal{C}^{-}\right)=56 x_{0}+60 x_{1}+60 x_{2}+60 x_{3}+56 x_{4}+60 x_{5}+60 x_{6}+60 x_{7}+56 x_{8} \quad \text { if } g=9, \\
& w_{T}\left(\mathcal{C}^{-}\right)=(7 g-12)\left(x_{0}+x_{2 k}\right)+(7 g-15) x_{k}+(9 g-18) \sum_{i \neq 0, k, 2 k} x_{i} \quad \text { if } g \geqslant 11, \\
& w_{T}\left(\mathcal{C}^{\star}\right)=\frac{15 g-29}{2}\left(x_{0}+x_{2 k}\right)+(8 g-16) x_{k}+(9 g-20) \sum_{i \neq 0, k, 2 k} x_{i} .
\end{aligned}
$$

Lemma 5.3. Suppose $g \geqslant 5$. Let $\mathcal{C}^{+}, \mathcal{C}^{-}$, and $\mathcal{C}^{\star}$ be the monomial bases of cosyzygies for $R$ constructed in Section 4. Then the convex hull of the T-states $w_{T}\left(\mathcal{C}^{+}\right), w_{T}\left(\mathcal{C}^{-}\right)$, and $w_{T}\left(\mathcal{C}^{\star}\right)$ contains the barycenter

$$
\frac{3(3 g-5)(g-1)}{g} \sum_{i=0}^{2 k} x_{i}
$$

Proof. Equivalently, we may show that the 0-state is an effective linear combination of $w_{T}\left(\mathcal{C}^{+}\right)$, $w_{T}\left(\mathcal{C}^{-}\right)$, and $w_{T}\left(\mathcal{C}^{\star}\right)$ modulo $\sum_{i=0}^{2 k} x_{i}$. First we deal with the low genus cases. We note that

$$
\begin{aligned}
w_{T}\left(\mathcal{C}^{+}\right) & =24 \sum_{i=0}^{2 k} x_{i} \quad \text { if } g=5, \\
w_{T}\left(\mathcal{C}^{+}\right)+6 w_{T}\left(\mathcal{C}^{-}\right) & =288 \sum_{i=0}^{2 k} x_{i} \quad \text { if } g=7, \\
w_{T}\left(\mathcal{C}^{+}\right)+8 w_{T}\left(\mathcal{C}^{-}\right) & =528 \sum_{i=0}^{2 k} x_{i} \quad \text { if } g=9 .
\end{aligned}
$$

Assuming $g \geqslant 11$, we have

$$
\begin{aligned}
& w_{T}\left(\mathcal{C}^{+}\right)=(g-5)(g-1)\left(x_{0}+x_{k}+x_{2 k}\right) \quad\left(\bmod \sum_{i=0}^{2 k} x_{i}\right), \\
& w_{T}\left(\mathcal{C}^{-}\right)=-(2 g-6)\left(x_{0}+x_{2 k}\right)-(2 g-3) x_{k} \quad\left(\bmod \sum_{i=0}^{2 k} x_{i}\right), \\
& w_{T}\left(\mathcal{C}^{\star}\right)=-\frac{3 g-11}{2}\left(x_{0}+x_{2 k}\right)-(g-4) x_{k} \quad\left(\bmod \sum_{i=0}^{2 k} x_{i}\right) .
\end{aligned}
$$




\section{A. Deopurkar, M. Fedorchuk and D. Swinarski}

Form a positive linear combination $L$ of the last two lines as follows:

$$
\begin{aligned}
L & :=6 w_{T}\left(\mathcal{C}^{\star}\right)+(g-3) w_{T}\left(\mathcal{C}^{-}\right) \\
& =-\left(2 g^{2}-3 g-15\right)\left(x_{0}+x_{k}+x_{2 k}\right) \quad\left(\bmod \sum_{i=0}^{2 k} x_{i}\right) .
\end{aligned}
$$

Plainly, the 0-state is a positive linear combination of $w_{T}\left(\mathcal{C}^{+}\right)$and $L$.

Having established that $\operatorname{CoSyz}_{1}(R)$ is well defined in Lemma 5.1, we are now ready to prove our main theorem.

Theorem 5.4. Let $g \geqslant 5$ be odd. The balanced canonical ribbon $R$ of genus $g$ has an $\mathrm{SL}_{g}$-semistable first syzygy point.

Proof. Because $\mathrm{H}^{0}\left(R, \omega_{R}\right)$ is a multiplicity-free representation of $\mathbb{G}_{m} \subset \operatorname{Aut}(R)$ by Proposition 3.1, it suffices to verify the semi-stability of $\operatorname{CoSyz}_{1}(R)$ with respect to the maximal torus $T$ acting diagonally on the distinguished basis $\left\{x_{0}, \ldots, x_{2 k}\right\}$ of $\mathrm{H}^{0}\left(R, \omega_{R}\right)$ described in Proposition 3.1. For a proof of this reduction, see [MS11, Proposition 4.7], [AFS13, Proposition 2.4], or [Lun75, Corollaire 2 and Remarque 1].

The non-zero Plücker coordinates of $\operatorname{CoSyz}_{1}(R)$ diagonalizing the action of $T$ are precisely the monomial bases of cosyzygies for $R$. The $T$-semi-stability of $\operatorname{CoSyz}_{1}(R)$ now follows from Lemma 5.3 and the Hilbert-Mumford numerical criterion.

Corollary 5.5 (Theorem 2.6). A general canonical curve of odd genus $g \geqslant 5$ has a semi-stable first syzygy point.

Proof. This follows from Theorem 5.4 and the fact that $R$ deforms to a smooth canonical curve [Fon93].

\section{Computer calculations}

For any given genus, the semi-stability of any syzygy point of the balanced ribbon can in principle be verified numerically by enumerating all the $T$-states and checking that their convex hull contains the trivial state. We did calculations in Macaulay2 and polymake [GS, GJ] that established the GIT semi-stability of the first syzygy point of the balanced ribbon for $g=7,9,11,13$ and the second syzygy point for $g=9,11$. (Computations for higher genera appear to be impracticable.) The main theorem of this paper (on first syzygies) and our calculations for second syzygies in small genus provide the first evidence for feasibility of Farkas and Keel's approach to constructing the canonical model of $\bar{M}_{g}$.

\section{ACKNOWLEDGEMENTS}

We learned the details of Farkas and Keel's idea to use syzygies as the means to construct the canonical model of $\bar{M}_{g}$ from a talk given by Gavril Farkas at the AIM workshop Log minimal model program for moduli spaces held in December 2012. This paper grew out of our attempt to implement the roadmap laid out in that talk. We are grateful to AIM for the opportunity to meet. The workshop participants of the working group on syzygies, among them David Jensen, Ian Morrison, Anand Patel, and the present authors, verified by a computer computation our main result for $g=7$. This computation motivated us to search for a proof in the general case. 


\section{TOWARD GIT STABILITy OF SYZYGIES OF CANONICAL CURVES}

\section{REFERENCES}

ACGH85 E. Arbarello, M. Cornalba, P. A. Griffiths, and J. Harris, Geometry of algebraic curves. Vol. I, Grundlehren math. Wiss., vol. 267 (Springer-Verlag, New York, 1985); http://dx.doi.org/ 10.1007/978-1-4757-5323-3.

AF11a M. Aprodu and G. Farkas, Green's conjecture for curves on arbitrary K3 surfaces, Compos. Math. 147 (2011), no. 3, 839-851; http://dx.doi.org/10.1112/S0010437X10005099.

AF11b Koszul cohomology and applications to moduli, in Grassmannians, Moduli Spaces and Vector Bundles, Clay Math. Proc., vol. 14 (Amer. Math. Soc., Providence, RI, 2011), $25-50$.

AFS13 J. Alper, M. Fedorchuk, and D.I. Smyth, Finite Hilbert stability of (bi)canonical curves, Invent. Math. 191 (2013), no. 3, 671-718; http://dx.doi.org/10.1007/ s00222-012-0403-6.

AFSvdW13 J. Alper, M. Fedorchuk, D. I. Smyth, and F. van der Wyck, Log minimal model program for the moduli space of stable curves: The second flip, arXiv:1308.1148.

BB11 E. Ballico and S. Brannetti, Notes on projective normality of reducible curves, Rend. Circ. Mat. Palermo (2) 60 (2011), no. 3, 371-384; http://dx.doi.org/10.1007/ s12215-011-0060-4.

BE95 D. Bayer and D. Eisenbud, Ribbons and their canonical embeddings, Trans. Amer. Math. Soc. 347 (1995), no. 3, 719-756; http://dx.doi.org/10.2307/2154871.

FJ13 M. Fedorchuk and D. Jensen, Stability of 2nd Hilbert points of canonical curves, Int. Math. Res. Not. IMRN 2013 (2013), no. 22, 5270-5287; http://dx.doi.org/10.1093/imrn/ rns204.

Fon93 L.-Y. Fong, Rational ribbons and deformation of hyperelliptic curves, J. Algebraic Geom. 2 (1993), no. 2, 295-307.

Gie77 D. Gieseker, Global moduli for surfaces of general type, Invent. Math. 43 (1977), no. 3, 233-282; http://dx.doi.org/10.1007/BF01390081.

Gie82 L Lectures on moduli of curves, Tata Inst. Fund. Res. Lectures on Math. and Physics, vol. 69 (Tata Inst. Fund. Res., Bombay, 1982).

GJ E. Gawrilow and M. Joswig, polymake: a framework for analyzing convex polytopes, Version 2.12, available at http://www.math.tu-berlin.de/polymake/.

Gre84 M.L. Green, Koszul cohomology and the geometry of projective varieties, J. Differential Geom. 19 (1984), no. 1, 125-171; http://projecteuclid.org/euclid.jdg/1214438426.

GS D. Grayson and M. Stillman, macaulay2: a software system for research in algebraic geometry, Version 1.6, available at http://www.math.uiuc.edu/Macaulay2/.

Has05 B. Hassett, Classical and minimal models of the moduli space of curves of genus two, in Geometric Methods in Algebra and Number Theory, Progr. Math., vol. 235 (Birkhäuser Boston, Boston, MA, 2005), 169-192, http://dx.doi.org/10.1007/0-8176-4417-2_8.

HH09 B. Hassett and D. Hyeon, Log canonical models for the moduli space of curves: The first divisorial contraction, Trans. Amer. Math. Soc. 361 (2009), no. 8, 4471-4489; http://dx. doi.org/10.1090/S0002-9947-09-04819-3.

HH13 L Log minimal model program for the moduli space of stable curves: the first flip, Ann. of Math. (2) 177 (2013), no. 3, 911-968; http://dx.doi.org/10.4007/annals.2013.177. 3.3.

KM09 S. L. Kleiman and R. V. Martins, The canonical model of a singular curve, Geom. Dedicata 139 (2009), 139-166; http://dx.doi.org/10.1007/s10711-008-9331-4.

Lun75 D. Luna, Adhérences d'orbite et invariants, Invent. Math. 29 (1975), no. 3, 231-238; http: //dx.doi.org/10.1007/BF01389851.

MS11 I. Morrison and D. Swinarski, Gröbner techniques for low-degree Hilbert stability, Exp. Math. 20 (2011), no. 1, 34-56; http://dx.doi.org/10.1080/10586458.2011.544577. 


\section{A. Deopurkar, M. Fedorchuk and D. Swinarski}

Sch91a F. O. Schreyer, A standard basis approach to syzygies of canonical curves, J. reine angew. Math. 421 (1991), 83-123; http://dx.doi.org/10.1515/crll.1991.421.83.

Sch91b D. Schubert, A new compactification of the moduli space of curves, Compos. Math. 78 (1991), no. 3, 297-313; http://www. numdam.org/item?id=CM_1991_-78_3_297_0.

Voi02 C. Voisin, Green's generic syzygy conjecture for curves of even genus lying on a K3 surface, J. Eur. Math. Soc. (JEMS) 4 (2002), no. 4, 363-404; http://dx.doi.org/10.1007/ s100970200042.

Voi05 Green's canonical syzygy conjecture for generic curves of odd genus, Compos. Math. 141 (2005), no. 5, 1163-1190; http://dx.doi.org/10.1112/S0010437X05001387.

Anand Deopurkar anandrd@math.columbia.edu

Department of Mathematics, Columbia University, 2990 Broadway, New York, NY 10027, USA

Maksym Fedorchuk maksym.fedorchuk@bc.edu

Department of Mathematics, Boston College, 140 Commonwealth Avenue, Chestnut Hill, MA 02467, USA

David Swinarski dswinarski@fordham.edu

Department of Mathematics, Fordham University, 113 W 60th Street, New York, NY 10023, USA 